\title{
Ultrasound-Guided Transversus Abdominis Plane Block by Using Bupivacaine Alone or When Mixed with Dexmedetomidine or Dexamethazone for Pain Relief Among Abdominal Hysterectomy Patients
}

\author{
Nareman Elhamamy*1 and Mohamed Gamal Elmawy ${ }^{2}$ \\ ${ }^{1}$ Department of Obstetrics and Gynecology, Tanta University, Egypt \\ ${ }^{2}$ Department of Anesthesia, Tanta University, Egypt
}

Submission: December 30, 2019 ; Published: January 13, 2020

*Corresponding author: Hossam M Abd-Elnaby, Department of Obstetrics and Gynecology, Zagazig University Hospital, Egypt

\begin{abstract}
Total abdominal hysterectomy (TAH) has been considered a major surgical procedure that results in substantial postoperative pain and discomfort. The abdominal wall incision has been considered the main cause of such pain. The Transversus Abdominis Plane (TAP) Block is a local anesthetic block used to provide analgesia to the anterior and lateral abdominal wall. were the first to describe this abdominal field block. They described an anatomical landmark technique and provided evidence of blockade to the mid/lower thoracic and upper lumbar spinal nerves as they travelled in the fascial plane between the transversus abdominis and internal oblique muscles .In our Randomized, double blind, controlled clinical study which done in which we compared between four groups of patients who undergo abdominal hysterectomy through Pfannenstiel incision below level of umbilicus under general anesthesia without TAP block in first group, with preoperative ultrasound guided TAP block with $20 \mathrm{ml}$ bupivacaine $0.25 \%$ /side only in second group, with TAP block with bupivacaine and addition of dexmedetomidine $0.8 \mathrm{mcg} / \mathrm{kg} / \mathrm{side}$ in third group and with TAP block with bupivacaine and addition of dexamethasone 8mg /side in last group.
\end{abstract}

Throughout the procedure HR, MAP, Spo2, ETCO2 recorded at time of skin incision, 5, 10, 15, 30, 60, 90min and at time of skin closure. Furthermore, the number of administered I.V. fentanyl doses intra-operative calculated. And in first $48 \mathrm{~h}$ after surgery MAP, HR, Spo2, VAS (at rest and on couphing), nausea and vomiting, sedation score recorded on admission to PACU, 2, 4, 8, 12, 18, 24, 36, 48h post- operatively. Total amount of morphine consumption and time to first request for analgesia (TFA) recorded. Furthermore, Complications of TAP also recorded. The goals of our study were to achieve postoperative satisfaction in patients who undergone abdominal hysterectomy by pain relief with minimal opioid consumption and to assess when dexmedetomidine or dexamethasone added to bupivacaine, can prolong the duration of TAP block, can achieve better local anesthesia conditions and provides better pain control postoperatively without any major side-effects and which is superior of them.

In our study, results showed that patients who received TAP block with $20 \mathrm{ml}$ bupivacaine on each side even without any additives had significantly reduced morphine consumption at $48 \mathrm{~h}$ post operatively than in patients who not received TAP block. Also, Pain scores at rest and on coughing were significantly lower in the TAP block group even without additives especially at PACU up to $4 \mathrm{~h}$ after surgery. The incidence of sedation and PONV were reduced in patients undergoing TAP blockade, and there were no complications related to the block. In our study, we perform TAP block before surgical incision and before induction of anesthesia to assess success of block either by pin prick or by cold sensation. Also, we noticed the analgesic effect of TAP block intra operatively by improvement of hemodynamic measurements like HR and MAP with significantly reduced total fentanyl consumption in TAP block group even without any additives. The current study showed that the addition of $8 \mathrm{mg}$ dexamethasone to $20 \mathrm{~mL}$ bupivacaine $0.25 \%$ for TAP block on both sides resulted in prolongation the analgesic effect of TAP block by prolongation TFA, by reducing TMC at 48h postoperatively and a significant reduction of VAS pain score over the postoperative $48 \mathrm{~h}$ at rest and on coughing than in TAP block group with bupivacaine only.

We noticed reducing both pain and vomiting in which the group of TAP block with dexamethasone did not complain of nausea nor vomiting post-operatively except $10 \%$ of patients at 8 hours and $30 \%$ at 24 hours who complained of nausea only and the difference between groups was highly statistically significant. In our study, showed not only the efficacy of TAP block in relieving postoperative pain after lower abdominal surgeries but also extended time of pain relief in dexmedetomidine group by dose of $0.8 \mathrm{ug} / \mathrm{kg} / \mathrm{side}$ in addition to bupivacaine more than that with other groups by assessment of VAS during rest and during cough. Also, the postoperative first request of morphine was delayed and total morphine consumption through $48 \mathrm{~h}$ was obviously reduced in dexmedetomidine group more than that with other groups. This study demonstrates also reduction for postoperative nausea and vomiting that resulted either from pain or from high doses of morphine consumption. We concluded that, TAP block as a part of a multimodal analgesia regimen provided better analgesia after abdominal hysterectomy operation with lesser incidence of opioid-related side effects (sedation or nausea) compared with systemic analgesia with morphine due to the lower dose of morphine. TAP block as multimodal postoperative analgesia might be an option to facilitate postoperative early ambulation 


\section{Introduction}

The international association for the study of pain (IASP) defines pain as "an unpleasant sensory and emotional experience associated with actual or potential tissue damage or described in terms of such damage" [1]. Total abdominal hysterectomy (TAH) has been considered a major surgical procedure that results in substantial postoperative pain and discomfort [2]. Inadequate control of postoperative pain leads to several unwanted adverse events ranging from patients' discomfort, prolonged immobilization to thromboembolic phenomenon and pulmonary complications [3]. The Transversus Abdominis Plane (TAP) Block is a local anesthetic block used to provide analgesia to the anterior and lateral abdominal wall [4]. were the first to describe this abdominal field block. They described an anatomical landmark technique and provided evidence of blockade to the mid/lower thoracic and upper lumbar spinal nerves as they travelled in the fascial plane between the transversus abdominis and internal oblique muscles. Hebbard et al., (2007) have subsequently described an ultrasound-guided approach to the TAP block [5]. Success with this block is dependent on correctly identifying the neuro-fascial plane between the internal oblique and the transversus abdominis muscles. Ultrasound-based studies have shown poor accuracy of blind abdominal wall injections [5]. Unfortunately, (TAP) block duration is limited to effect of administered local anesthetics (LA). Recently, adjuvant medications were added to LA to prolong the effect of (TAP) block [6]. Dexamethasone has a long and efficient glucocorticoid structure which offers anti-inflammatory properties. When added to local anesthetics as an adjuvant in peripheral blocks, it increases the action time. It also prolongs the analgesia time contribute to the (TAP) Block [7].

\section{Aim of the Work}

The goals of our study were to achieve postoperative satisfaction in patients who undergone abdominal hysterectomy by pain relief with minimal opioid consumption and to assess when dexmedetomidine or dexamethasone added to bupivacaine, can prolong the duration of TAP block, can achieve better local anesthesia conditions and provides better pain control postoperatively without any major side-effects and which is superior of them.

\section{Patients and Methods}

The study was randomized control clinical trial conducted from January 2017 to august 2019 at the Obstetrics and Gynecology Department, Tanta University, Egypt. Eighty patients were enrolled and randomly assigned between the four groups; no refusals to participate. The patients were divided into four groups by randomization using computerized simple random then a nurse or a third person put serial numbers of patients inside closed envelopes.
The four groups are:

a) Group I ( $\mathrm{n}=20)$ that undergo the standard general anesthesia with TAP block with $22 \mathrm{ml}$ saline $0.9 \%$ per side as placebo group.

b) Group II $(\mathrm{n}=20)$ patients undergo standard general anesthesia with TAP block with $20 \mathrm{ml}$ of $0.25 \%$ bupivacaine + $2 \mathrm{ml}$ saline $0.9 \%$ per side.

c) Group III $(\mathrm{n}=20)$ patients undergo standard general anesthesia with TAP block with $20 \mathrm{ml} 0.25 \%$ bupivacaine + dexmedetomidine $0.8 \mathrm{mcg} / \mathrm{kg}$ dissolved in $2 \mathrm{ml}$ of normal saline $0.9 \%$ per side.

d) Group IV $(\mathrm{n}=20)$ patients undergo standard general anesthesia with TAP block with $20 \mathrm{ml}$ of $0.25 \%$ bupivacaine + $2 \mathrm{ml}$ dexamethasone (8mg) per side.

\section{Inclusion criteria}

a) American Society of Anesthesiology patient classification physical status I-II undergoing elective open abdominal hysterectomy through Pfannenstiel incision were allocated randomly to one of four groups.

b) Age range between (40-65) years.

c) Body mass index $(18-35) \mathrm{kg} / \mathrm{m}^{2}$.

\section{Exclusion criteria}

Patients unable or unwilling to give informed consent, those who had a history of relevant drug allergy, patients currently using analgesics or who had current acute or chronic pain or were receiving medical therapies considered to result in tolerance to opioids, patients taking other medications with $\alpha$-adrenergic blocking effect, hepatic or renal insufficiency, morbid obesity, local infection at the site of the block and blood coagulation pathologies.

\section{Withdrawal criteria}

Cases that were missed during the study or failed technique were excluded.

\section{Parameters Used to Evaluate the Study}

\section{Intraoperative}

Throughout the procedure HR, MAP, Sp02, EtCo2 were recorded at time of skin incision, 5, 10, 15, 30, 60, 90 and at time of skin closure. Furthermore, the number of administered I.V. fentanyl doses intra-operative were calculated.

\section{Postoperative}

Postoperatively and in first 48h MAP, HR, Spo 2, VAS (at rest and on coughing), nausea and vomiting, sedation score were recorded on admission to PACU or ward, 2, 4, 8, 12, 18, 24, 36, 48h postoperatively by an observer who was unaware of the study protocol. 
Total amount of morphine consumption and time to first request for analgesia (TFA) were recorded. Furthermore, Complications of TAP were recorded which may include failure of the block, intramuscular hematoma and abscess, visceral puncture or perforation. Undesirable effects from opioid use as postoperative nausea and vomiting (PONV), sedation and respiratory depression were monitored and recorded for all groups.

Pain scores observed and recorded using the pain assessment on a $100-\mathrm{mm}$ line (visual analogue scale (VAS); $0=$ no pain, $100=$ worst pain imaginable) both at rest and on coughing. PONV measured using a categorical scoring system (None $=0$; nausea $=1$; retching $=2$; vomiting $=3$ ). Detection of nausea occurred when score $>0$ at any time point postoperatively which managed by IV injection of $10 \mathrm{mg}$ metoclopramide.

Sedation scores were rated using a sedation scale (Awake and alert=0; quietly awake $=1$; asleep but easily roused=2; deep sleep=3). Sedation is defined as a sedation score $>0$ at any postoperative time point. Respiratory depression is defined as respiratory rate $<8$ breaths per min and / or $\mathrm{SpO} 2<90 \%$ on the room air.

\section{Methods}

The study protocol was approved by the Obstetrics and Gynecology Department scientific and ethical committees. All patients were informed about the study design and objectives as well as tools and techniques. Informed consent had been signed by every patient prior to the study.

\section{Randomization}

A computer-generated table into one of four study groups randomly allocated patients. A sealed envelope with the study medication to be injected will be given to the research coordinator on the morning of surgery. Both the research coordinator and the physician performing the block will be blinded to the injected drug.

\section{Preoperative day}

All participating patients were interviewed preoperatively during their preoperative clearance appointment. The goal and endpoints of the study were discussed, and their consents were taken. Understanding of the Visual Analog Score for pain (VAS) were reviewed and emphasized. On physical examination, special attention given to document normal sensation at the site of the upcoming hysterectomy surgery where the TAP block effect take place. Routine preoperative assessment was done to all patients including history, clinical examination, laboratory investigations (complete blood picture, kidney function tests, liver function tests, prothrombin time, partial thromboplastin time), chest X-ray, electrocardiogram [ECG] was done for patients above 40 years old.

\section{Operative day}

Performance of transversus abdominis plane block technique: All patients will be brought to the preanesthesia block room for placement of the block $30 \mathrm{~min}$ pre-operatively. Standard ASA monitors will be placed; pulse oximetry, non-invasive blood pressure cuff and ECG. Sedation with midazolam (2-4mg IV) with running 500cc ringer solution will be administered. The patients' vital signs and conscious status will be monitored and recorded throughout the procedure by the conscious sedation nurses The TAP block was performed using ultrasound machine (SonoScape) model. the scanning probe was the linear multi-frequency 11-5 MHz transducer (L746 11-5 MHz Linear Array)

Equipment and technique: Ultrasound machine was used for all blocks. The blocks performed using 22 -gauge $(100 \mathrm{~mm})$ needles. All blocks were performed with the patients in the supine position; the skin at the site of the block was prepped with antiseptic solution. Strict aseptic technique used to include sterile gloves, masks, overhead caps, sterile drapes and sterile ultrasound probe covers. The ultrasound probe was placed in the midaxillary line of each side, midway between the lower costal margin and the iliac crest. Scanning to appreciate the three muscular layers forming the abdominal wall; from superficial to deep; external oblique, internal oblique and transversus abdominis. Special care was taken to identify the potential plane between the internal oblique muscle and the transversus abdominis and differentiate it from the deeper fascia transversalis that separate the muscles from the preperitoneal fat and Peritoneum. The site of the needle entry was injected with lidocaine $1 \%$. The needle was inserted in plane until its tip located in between the internal oblique and transversus abdominis muscles then $2 \mathrm{ml}$ of epinephrine $1 / 200,000$ diluted in $5 \mathrm{~mL}$ normal saline were injected to widen the neurofascial plane and, exclude intravascular injection. After that negative aspiration was done, a $22 \mathrm{ml}$ of the study solution according to the type of group as mentioned before injected in small increments over few minutes observing the spread dissection of the fluid between the two appropriate muscles. Patients were monitored for any cardiovascular signs of local anesthetic toxicity during the injection. So 'intralipid', a commonly available intravenous lipid emulsion, can be effective in treating severe cardiotoxicity secondary to local anesthetic overdose. Then patients transported to the operating room after the block.

General anesthesia technique: $0 \mathrm{n}$ arrival to the operative room after establishing a peripheral intravenous access, patients were given ranitidine (50mg, IV), and metoclopramide (10mg, IV). After standard monitoring including ECG, noninvasive blood pressure and pulse oximetry were connected to patient preoxygenation for 5 minutes done General anesthesia was standardized for all patients in four groups. Fentanyl $2 \mathrm{mcg} / \mathrm{kg}$, propofol $2 \mathrm{mg} / \mathrm{kg}$ was intravenously administered and cisatracurium $0.1 \mathrm{mg} / \mathrm{kg}$ was 
given to facilitate tracheal intubation. Endotracheal tube with suitable size used to intubate the trachea. Lungs were ventilated by pressure-controlled mode to maintain normocapnia ETCO2 around 35-38 mmHg and to maintain 02 saturation > 98\%. Isoflurane/02 mixture was administered. Concentration of the inhalational agent titrated to adequate depth of anesthesia guided by the clinical judgment of the anesthesiologist and recorded (1-1.5 volume \%). Fentanyl $0.5 \mathrm{mcg} / \mathrm{kg}$ IV administered for any intraoperative increase in the HR or mean arterial pressure (MAP) above $20 \%$ of baseline. Amount of fluids needed calculated to each patient and given throughout the operation with consideration of blood loss replacement. After Surgery, patients were taken to PACU (Post Anesthesia Care Unit). Nurses in PACU were also blinded to the type of medication used in the TAP block. PACU nurses moved the patients from PACU to ward when they meet the standard criteria (modified Aldrete score of 9 or more) (table 1).

Table 1: Modified Aldrete scoring system.

\begin{tabular}{|c|c|c|}
\hline Criteria & Ability & Score \\
\hline \multirow{3}{*}{ Level of consciousness } & Awake and oriented & 2 \\
\hline & Arousable with minimal stimulation & 1 \\
\hline & Responsive only to tactile stimulation & 0 \\
\hline \multirow{3}{*}{ Physical activity } & Able to move all extremities on command & 2 \\
\hline & Some weakness in movement of extremities & 1 \\
\hline & Unable to voluntarily move extremities & 0 \\
\hline \multirow{3}{*}{ Hemodynamic stability } & Blood pressure $<15 \%$ of baseline MAP (mean arterial pressure) value & 2 \\
\hline & Blood pressure $15 \%-30 \%$ of baseline MAP value & 1 \\
\hline & Blood pressure $>30 \%$ below baseline MAP value & 0 \\
\hline \multirow{3}{*}{ Respiratory stability } & Able to breathe deeply & 2 \\
\hline & Tachypnea with good cough & 1 \\
\hline & Dyspneic with weak cough & 0 \\
\hline \multirow{3}{*}{ Oxygen saturation status } & Maintains value $>90 \%$ on room air & 2 \\
\hline & Requires supplemental oxygen (nasal prongs) & 1 \\
\hline & Saturation $<90 \%$ with supplemental oxygen & 0 \\
\hline \multirow{3}{*}{ Postoperative pain assessment } & None or mild discomfort & 2 \\
\hline & Moderate to severe pain controlled with IV analgesics & 1 \\
\hline & Persistent severe pain & 0 \\
\hline \multirow{3}{*}{ Postoperative emetic symptoms } & None or mild nausea with no active Vomiting & 2 \\
\hline & Transient vomiting or retching & 1 \\
\hline & Persistent moderate to severe nausea and vomiting & 0 \\
\hline Total Score & & 14 \\
\hline
\end{tabular}

All patients enrolled in the study were kept for 48 hours stay. All patients were given bolus of morphine $0.04 \mathrm{mg} / \mathrm{kg}$, acetaminophen $1 \mathrm{~g}$ IV every $6 \mathrm{~h}$ during first $24 \mathrm{~h}$ after surgery and voltaren IM every $12 \mathrm{~h}$ in first $24 \mathrm{hs}$ then Acetaminophen $1 \mathrm{~g}$ was given orally every $6 \mathrm{~h}$ for next 4 days. Patients were given IV boluses of $0.02 \mathrm{mg} / \mathrm{kg}$ morphine when needed when visual analog scale (VAS) score with cough $>30 \mathrm{~mm}$ or on patient demand. $10 \mathrm{mg}$ metoclopramide IV was administered in case of reported nausea and/or vomiting.

\section{Statistical Analysis}

The collected data were tabulated and analyzed using SPSS version 16 software (SpssInc, Chicago, ILL Company) Categorical data were presented as number and percentages while quantitative data were expressed as mean \pm standard deviation, and range.
Chi square test $\left(\mathrm{X}^{2}\right)$ was used to analyze categorical variables. Quantitative data were tested for normality using Kolomogrov Smirnove test, assuming normality at $\mathrm{P}>0.05$, using analysis of variance ANOFA (F test) with post Hoc test for normally distributed quantitative variables. The accepted level of significance in this work was stated at $0.05(\mathrm{P}<0.05$ was considered significant $)$.

$P$ value $>0.05$ is non-significant (NS)

$\mathrm{P}<0.05$ is significant $(\mathrm{S})$

$\mathrm{P} \leq 0.001$ is highly significant (HS).

The previous measurements were recorded intraoperatively and during the first 48 hours postoperatively. The primary aim of the work was to study the clinical utility of transversus abdominis plane block compared with general anesthesia alone 
and see how it could be superior to conventional analgesia for patient undergoing abdominal hysterectomy. Secondary outcome measures the effect of dexmedetomidine and dexamethasone on the time to the first request for additional analgesics (TFA) and which is superior of them to prolong the duration of TAP block.

Result

The study was conducted from January 2017 to august 2019 at the Obstetrics and Gynecology Department, Tanta University, Egypt. Eighty patients were enrolled and randomly assigned between the four groups; no refusals to participate. All patients completed the study protocol. Demographic data (age and body mass index) of the patients, the duration of surgical procedure and the duration of anesthesia are demonstrated in Table 2. Table 2 shows that the four groups were matched as regard age, BMI, anesthesia and surgical duration and the difference was statistically not significant. Table 3 shows a highly statistically significant difference between the four groups regarding intraoperative HR recorded at skin incision, 5, 10, 15, 30, 60, 90 minutes and at skin closure. While pre-operative HR was nearly the same among patients of the four groups. Table 4 shows a highly statistically significant difference between the four groups regarding intra-operative MAP recorded at skin incision, 5, 10, 15, 30, 60, 90 minutes and at skin closure. While pre-operative MAP was nearly the same among patients of the four groups.

Table 2: Demographic data and clinical characteristics of the studied cases.

\begin{tabular}{|c|c|c|c|c|c|c|}
\hline Variables & $\begin{array}{c}\text { Group I } \\
\mathbf{N}=20\end{array}$ & $\begin{array}{c}\text { Group II } \\
\mathbf{N}=\mathbf{2 0}\end{array}$ & $\begin{array}{c}\text { Group III } \\
\quad \mathbf{N}=\mathbf{2 0}\end{array}$ & $\begin{array}{l}\text { Group IV } \\
\quad \mathrm{N}=\mathbf{2 0}\end{array}$ & F test & P-value \\
\hline $\begin{array}{l}\text { Age/years } \\
\text { Mean } \pm \text { SD } \\
\text { Range }\end{array}$ & $\begin{array}{l}49.8 \pm 6.63 \\
40-58\end{array}$ & $\begin{array}{l}49.8 \pm 5.63 \\
40-62\end{array}$ & $\begin{array}{l}50.4 \pm 6.82 \\
\quad 40-62\end{array}$ & $\begin{array}{c}50.3 \pm 5.36 \\
40-62\end{array}$ & 0.055 & 0.983 \\
\hline $\begin{array}{c}\text { BMI } \\
\text { Mean } \pm \text { SD } \\
\text { Rang }\end{array}$ & $\begin{array}{c}31.4 \pm 1.44 \\
27-34.6\end{array}$ & $\begin{array}{c}31.7 \pm 1.71 \\
27-34.4\end{array}$ & $\begin{array}{c}31.4 \pm 1.89 \\
27-34.4\end{array}$ & $\begin{array}{c}31.2 \pm 2.01 \\
27-34.4\end{array}$ & 2.86 & 0.04 \\
\hline $\begin{array}{c}\text { Anesthesia duration } \backslash \text { min } \\
\text { Mean } \pm \text { SD } \\
\text { Range }\end{array}$ & $\begin{array}{c}119 \pm 12.31 \\
105-140\end{array}$ & $\begin{array}{c}109.8 \pm 16.5 \\
85-140\end{array}$ & $\begin{array}{c}109.5 \pm 14.5 \\
80-140\end{array}$ & $\begin{array}{c}111.8 \pm 17.6 \\
85-140\end{array}$ & 1.68 & 0.178 \\
\hline $\begin{array}{l}\text { Surgical duration } \backslash \text { min } \\
\text { Mean } \pm S D \\
\text { Range }\end{array}$ & $\begin{array}{c}94 \pm 16.4 \\
75-120\end{array}$ & $\begin{array}{c}89.5 \pm 17.2 \\
70-120\end{array}$ & $\begin{array}{c}89.8 \pm 15.3 \\
70-120\end{array}$ & $\begin{array}{c}95 \pm 16.7 \\
70-120\end{array}$ & 0.602 & 0.616 \\
\hline
\end{tabular}

Table 3: Pre-operative and intra-operative Heart Rate (HR) among the studied groups.

\begin{tabular}{|c|c|c|c|c|c|c|}
\hline Variables & $\begin{array}{l}\text { Group I } \\
\mathrm{N}=20\end{array}$ & $\begin{array}{l}\text { Group II } \\
\mathrm{N}=20\end{array}$ & $\begin{array}{l}\text { Group III } \\
\mathbf{N}=\mathbf{2 0}\end{array}$ & $\begin{array}{l}\text { Group IV } \\
\mathrm{N}=\mathbf{2 0}\end{array}$ & F test & P-value \\
\hline $\begin{array}{l}\text { Pre-operative basal HR } \\
\text { Mean } \pm \text { SDRange }\end{array}$ & $\begin{array}{l}79.4 \pm 6.9 \\
70-90\end{array}$ & $\begin{array}{c}75.5 \pm 5.5 \\
66-82\end{array}$ & $\begin{array}{c}75.5 \pm 5.5 \\
66-82\end{array}$ & $\begin{array}{l}75.5 \pm 5.5 \\
66-82\end{array}$ & 2.23 & 0.09 \\
\hline $\begin{array}{l}\text { Intra-operative HR at skin incision } \\
\text { Mean } \pm \text { SD Rang }\end{array}$ & $\begin{array}{c}104.8 \pm 12.6 \\
88-120\end{array}$ & $\begin{array}{c}81.1 \pm 5.98 \\
70-90\end{array}$ & $\begin{array}{c}80 \pm 6.1 \\
70-90\end{array}$ & $\begin{array}{c}81.4 \pm 8.9 \\
65-92\end{array}$ & 37.02 & $<0.001$ \\
\hline $\begin{array}{l}\text { Intra-operative } \mathrm{HR} \text { at } 5 \mathrm{~min} \\
\text { Mean } \pm \text { SD Range }\end{array}$ & $\begin{array}{c}94.6 \pm 15.4 \\
75-114\end{array}$ & $\begin{array}{c}76.5 \pm 7.4 \\
62-90\end{array}$ & $\begin{array}{c}69.7 \pm 3.7 \\
60-78\end{array}$ & $\begin{array}{c}76.7 \pm 7.5 \\
62-90\end{array}$ & 25.16 & $<0.001$ \\
\hline $\begin{array}{c}\text { Intra-operative HR at } 10 \mathrm{~min} \\
\text { Mean } \pm \text { SD Range }\end{array}$ & $\begin{array}{c}101.2 \pm 21.9 \\
78-139\end{array}$ & $\begin{array}{c}77.3 \pm 8.2 \\
60-88\end{array}$ & $\begin{array}{c}66.3 \pm 3.2 \\
60-72\end{array}$ & $\begin{array}{l}77.6 \pm 8.6 \\
60-88\end{array}$ & 27.4 & $<0.001$ \\
\hline $\begin{array}{c}\text { Intra-operative } \mathrm{HR} \text { at } 15 \mathrm{~min} \\
\text { Mean } \pm \text { SD Range }\end{array}$ & $\begin{array}{c}107.6 \pm 16.9 \\
77-131\end{array}$ & $\begin{array}{c}72.95 \pm 6.3 \\
60-84\end{array}$ & $\begin{array}{c}63.9 \pm 3.04 \\
59-70\end{array}$ & $\begin{array}{l}72.5 \pm 5.9 \\
60-84\end{array}$ & 62.16 & $<0.001$ \\
\hline $\begin{array}{c}\text { Intra-operative } \mathrm{HR} \text { at } 30 \mathrm{~min} \\
\text { Mean } \pm S D \text { Range }\end{array}$ & $\begin{array}{c}100.4 \pm 7.1 \\
90-110\end{array}$ & $\begin{array}{c}77.95 \pm 7.6 \\
66-92\end{array}$ & $\begin{array}{c}62.5 \pm 3.1 \\
60.69\end{array}$ & $\begin{array}{c}78.6 \pm 8.1 \\
66-92\end{array}$ & 104.8 & $<0.001$ \\
\hline $\begin{array}{c}\text { Intra-operative } \mathrm{HR} \text { at } 60 \mathrm{~min} \\
\text { Mean } \pm \mathrm{SD} \text { Range }\end{array}$ & $\begin{array}{c}88 \pm 7.8 \\
75-98\end{array}$ & $\begin{array}{c}78.3 \pm 5.98 \\
66-89\end{array}$ & $\begin{array}{c}63 \pm 3.4 \\
60-70\end{array}$ & $\begin{array}{c}78.7 \pm 6.4 \\
66-89\end{array}$ & 57.51 & $<0.001$ \\
\hline $\begin{array}{c}\text { Intra-operative } \mathrm{HR} \text { at } 90 \mathrm{~min} \\
\text { Mean } \pm \text { SD Range }\end{array}$ & $\begin{array}{c}89 \pm 7.6 \\
79-96\end{array}$ & $\begin{array}{c}82.1 \pm 6.9 \\
73-93\end{array}$ & $\begin{array}{c}63 \pm 3.4 \\
59-68\end{array}$ & $\begin{array}{c}83.8 \pm 7.9 \\
73-93\end{array}$ & 28.79 & $<0.001$ \\
\hline $\begin{array}{l}\text { Intra-operative HR at skin closure } \\
\text { Mean } \pm \text { SD Range }\end{array}$ & $\begin{array}{l}79 \pm 6.4 \\
71-90\end{array}$ & $\begin{array}{c}76.7 \pm 7.5 \\
62-90\end{array}$ & $\begin{array}{c}63.8 \pm 3.5 \\
59-70\end{array}$ & $\begin{array}{c}82.3 \pm 9.96 \\
65-96\end{array}$ & 25.02 & $<0.001$ \\
\hline
\end{tabular}


Table 4: Pre-operative and intra-operative Mean Arterial blood pressure (MAP) among the studied groups.

\begin{tabular}{|c|c|c|c|c|c|c|}
\hline Variables & $\begin{array}{c}\text { Group I } \\
\mathbf{N}=\mathbf{2 0}\end{array}$ & $\begin{array}{l}\text { Group II } \\
\mathrm{N}=20\end{array}$ & $\begin{array}{l}\text { Group III } \\
\mathrm{N}=20\end{array}$ & $\begin{array}{c}\text { Group IV } \\
\mathrm{N}=\mathbf{2 0}\end{array}$ & F test & P-value \\
\hline $\begin{array}{l}\text { Pre-operative basal MAP } \\
\text { Mean } \pm \text { SD Range }\end{array}$ & $80.8 \pm 3.878-88$ & $81.8 \pm 8.476-88$ & $80.7 \pm 6.177-87$ & $\begin{array}{c}81.7 \pm 8.6 \\
76-87\end{array}$ & 1.23 & 0.213 \\
\hline $\begin{array}{l}\text { Intra-operative MAP at skin incision } \\
\text { Mean } \pm \text { SD Rang }\end{array}$ & $\begin{array}{l}89.7 \pm 6.04 \\
82-99\end{array}$ & $\begin{array}{c}72 \pm 8.6 \\
60-90\end{array}$ & $\begin{array}{l}70.4 \pm 6.98 \\
60-90\end{array}$ & $\begin{array}{c}78.6 \pm 9.1 \\
62-94\end{array}$ & 25.2 & $<0.001$ \\
\hline $\begin{array}{l}\text { Intra-operative MAP at } 5 \mathrm{~min} \\
\text { Mean } \pm \text { SD Range }\end{array}$ & $\begin{array}{c}88.8 \pm 8.6 \\
78-99\end{array}$ & $\begin{array}{c}69.1 \pm 6.3 \\
58-86\end{array}$ & $\begin{array}{c}63.5 \pm 6.2 \\
55-80\end{array}$ & $\begin{array}{c}72.5 \pm 7.5 \\
58-86\end{array}$ & 45.16 & $<0.001$ \\
\hline $\begin{array}{l}\text { Intra-operative MAPat10min } \\
\text { Mean } \pm \text { SD Range }\end{array}$ & $\begin{array}{c}87.2 \pm 9.3 \\
70-94\end{array}$ & $\begin{array}{c}69.1 \pm 7.3 \\
57-82\end{array}$ & $\begin{array}{c}61.3 \pm 4.4 \\
55-72\end{array}$ & $\begin{array}{c}69.9 \pm 8.5 \\
57-84\end{array}$ & 41.74 & $<0.001$ \\
\hline $\begin{array}{l}\text { Intra-operative MAPat15min } \\
\text { Mean } \pm \text { SD Range }\end{array}$ & $\begin{array}{c}86.8 \pm 8.3 \\
74-99\end{array}$ & $\begin{array}{c}70.7 \pm 6.4 \\
62-88\end{array}$ & $\begin{array}{c}62.9 \pm 3.1 \\
60-70\end{array}$ & $\begin{array}{c}69.7 \pm 5.7 \\
62-77\end{array}$ & 54.33 & $<0.001$ \\
\hline $\begin{array}{l}\text { Intra-operative MAPat30min } \\
\text { Mean } \pm \text { SD Range }\end{array}$ & $\begin{array}{c}85.8 \pm 4.4 \\
80-91\end{array}$ & $\begin{array}{l}72.2 \pm 11.5 \\
55-94\end{array}$ & $\begin{array}{l}59.9 \pm 2.6 \\
55-67\end{array}$ & $\begin{array}{c}65.8 \pm 6.1 \\
55-76\end{array}$ & 49.99 & $<0.001$ \\
\hline $\begin{array}{l}\text { Intra-operative MAPat60min } \\
\text { Mean } \pm \text { SD Range }\end{array}$ & $\begin{array}{c}85.6 \pm 4.1 \\
78-89\end{array}$ & $\begin{array}{c}69.9 \pm 7.6 \\
58-88\end{array}$ & $\begin{array}{c}61.6 \pm 4.4 \\
55-68\end{array}$ & $\begin{array}{c}66.9 \pm 4.9 \\
58-72\end{array}$ & 72.95 & $<0.001$ \\
\hline $\begin{array}{l}\text { Intra-operative MAPat90min } \\
\text { Mean } \pm \text { SD Range }\end{array}$ & $\begin{array}{l}81 \pm 2.6 \\
78-84\end{array}$ & $\begin{array}{l}71.8 \pm 7.8 \\
57-84\end{array}$ & $\begin{array}{l}63.2 \pm 4.9 \\
57-72\end{array}$ & $\begin{array}{c}67 \pm 5.1 \\
57-73 \\
\end{array}$ & 24.84 & $<0.001$ \\
\hline $\begin{array}{c}\text { Intra-operative MAP at skin closure } \\
\text { Mean } \pm \text { SD } \\
\text { Range }\end{array}$ & $\begin{array}{l}80.8 \pm 3.97 \\
75-86\end{array}$ & $\begin{array}{c}72.7 \pm 8.4 \\
62-88\end{array}$ & $\begin{array}{c}67.7 \pm 6.1 \\
60-88\end{array}$ & $\begin{array}{c}73.7 \pm 8.6 \\
62-88\end{array}$ & 11.44 & $<0.001$ \\
\hline
\end{tabular}

Table 5: Intra-operative Pulsed Oxygen Saturation $\left(\mathrm{SPO}_{2}\right)$ among the studied groups.

\begin{tabular}{|c|c|c|c|c|c|c|}
\hline Variables & $\begin{array}{c}\text { Group I } \\
\mathrm{N}=20\end{array}$ & $\begin{array}{c}\text { Group II } \\
\mathrm{N}=20\end{array}$ & $\begin{array}{c}\text { Group III } \\
\mathrm{N}=20\end{array}$ & $\begin{array}{c}\text { Group IV } \\
\mathrm{N}=20\end{array}$ & $F$ test & P-value \\
\hline $\begin{array}{c}\text { Intra-operative } \mathrm{SPO}_{2} \text { at skin incision } \\
\text { Mean } \pm \mathrm{SD} \\
\text { Rang }\end{array}$ & $\begin{array}{l}99.2 \pm 0.77 \\
98-100\end{array}$ & $\begin{array}{c}98.5 \pm 0.61 \\
98-100\end{array}$ & $\begin{array}{l}98.5 \pm 0.68 \\
98-100\end{array}$ & $\begin{array}{c}98.9 \pm 0.91 \\
98-100\end{array}$ & 2.12 & 0.121 \\
\hline $\begin{array}{c}\text { Intra-operative } \mathrm{SPO}_{2} \text { at } 5 \mathrm{~min} \\
\text { Mean } \pm \mathrm{SD} \\
\text { Range }\end{array}$ & $\begin{array}{c}99.3 \pm 0.65 \\
98-100\end{array}$ & $\begin{array}{c}98.5 \pm 0.69 \\
98-100\end{array}$ & $\begin{array}{l}98.5 \pm 0.69 \\
98-100\end{array}$ & $\begin{array}{c}98.8 \pm 0.95 \\
98-100\end{array}$ & 2.16 & 0.08 \\
\hline $\begin{array}{c}\text { Intra-operative } \mathrm{SPO}_{2} \text { at } 10 \mathrm{~min} \\
\text { Mean } \pm \mathrm{SD} \text { Range }\end{array}$ & $\begin{array}{c}99.5 \pm 0.65 \\
98-100\end{array}$ & $\begin{array}{c}98.4 \pm 0.58 \\
98-100\end{array}$ & $\begin{array}{l}98.5 \pm 0.69 \\
98-100\end{array}$ & $\begin{array}{c}98.9 \pm 0.95 \\
98-100\end{array}$ & 2.24 & 0.09 \\
\hline $\begin{array}{c}\text { Intra-operative } \mathrm{SPO}_{2} \text { at } 15 \mathrm{~min} \\
\text { Mean } \pm \mathrm{SD} \text { Range }\end{array}$ & $\begin{array}{l}99.2 \pm 0.65 \\
98-100\end{array}$ & $\begin{array}{l}98.3 \pm 0.47 \\
98-99\end{array}$ & $\begin{array}{l}98.4 \pm 0.49 \\
98-99\end{array}$ & $\begin{array}{c}98.5 \pm 0.61 \\
98-100\end{array}$ & 1.55 & 0.111 \\
\hline $\begin{array}{c}\text { Intra-operative } \mathrm{SPO}_{2} \text { at } 30 \mathrm{~min} \\
\text { Mean } \pm \mathrm{SD} \text { Range }\end{array}$ & $\begin{array}{l}99.3 \pm 0.65 \\
98-100\end{array}$ & $\begin{array}{c}98.4 \pm 0.59 \\
98-100\end{array}$ & $\begin{array}{l}98.5 \pm 0.69 \\
98-100\end{array}$ & $\begin{array}{c}98.8 \pm 0.86 \\
98-100\end{array}$ & 1.39 & 0.09 \\
\hline $\begin{array}{c}\text { Intra-operative } \mathrm{SPO}_{2} \text { at } 60 \mathrm{~min} \\
\text { Mean } \pm \mathrm{SD} \text { Range }\end{array}$ & $\begin{array}{c}99.3 \pm .65 \\
98-100\end{array}$ & $\begin{array}{c}98.6 \pm 0.76 \\
98-100\end{array}$ & $\begin{array}{l}98.6 \pm 0.76 \\
98-100\end{array}$ & $\begin{array}{c}98.9 \pm 0.93 \\
98-100\end{array}$ & 1.65 & 0.189 \\
\hline $\begin{array}{c}\text { Intra-operative } \mathrm{SPO}_{2} \text { at } 90 \text { min } \\
\text { Mean } \pm \mathrm{SD} \text { Range }\end{array}$ & $\begin{array}{l}98.7 \pm 0.49 \\
98-99\end{array}$ & $\begin{array}{l}98.8 \pm 0.67 \\
98-99\end{array}$ & $\begin{array}{l}98.9 \pm 0.74 \\
98-100\end{array}$ & $\begin{array}{c}99.1 \pm 0.41 \\
98-100\end{array}$ & 1.08 & 0.369 \\
\hline $\begin{array}{c}\text { Intra-operative } \mathrm{SPO}_{2} \text { at skin closure Mean } \pm \mathrm{SD} \\
\text { Range }\end{array}$ & $\begin{array}{l}98.8 \pm 0.41 \\
98-99\end{array}$ & $\begin{array}{c}99.1 \pm 0.61 \\
98-100\end{array}$ & $\begin{array}{c}99.1 \pm 0.55 \\
98-100\end{array}$ & $\begin{array}{c}99.3 \pm 0.57 \\
98-100\end{array}$ & 2.14 & 0.105 \\
\hline
\end{tabular}

Table 5 shows no statistically significant difference between the four groups regarding intra-operative SPO2 recorded at skin incision, 5, 10, 15, 30, 60, 90 minutes and at skin closure. Table 6 shows no statistically significant difference between the four groups regarding intra-operative ETCO2 recorded at skin incision, $5,10,15,30,60,90$ minutes and at skin closure. As Table 7 shows a highly statistically significant difference between the four groups regarding intra-operative Total fentanyl consumption (TFC) at skin incision, 5, 10, 15, 30, 60, 90 minutes and at skin closure. As Table 8 shows a highly statistically significant difference between the four groups regarding post-operative HR recorded at PACU, $2,4,8,12,18,24$ and 36 hours. While HR recorded at 48 hours post-operatively was close among patients of four groups and the difference did not reach significant level. As Table 9 shows a 
highly statistically significant difference between the four groups regarding post-operative MAP recorded at PACU, 2, 4, 8, 12, 18, 24, 36 and 48 hours. As Table 10 shows a highly statistically significant difference between the four groups regarding post-operative SPO2 recorded at PACU, 2, 4, 8, 12, 18, 24, 36 and 48 hours. As Table 11 shows a highly statistically significant difference between the four groups regarding post-operative VAS score at rest recorded at PACU, 2, 4, 8, 12, 18, 24, 36 and 48 hours. As Table $12 \& 13$ shows a highly statistically significant difference between the four groups regarding post-operative VAS score on coughing recorded at PACU, 2, 4, 8, 12, 18, 24, 36 and 48 hours. As Table 14 shows that patients of group IV did not complain of nausea nor vomiting post-operatively except $10 \%$ of patients at 8 hours and $30 \%$ at 24 hours who complained of nausea only, and the difference between groups was highly statistically significant. While among group III there was $15 \%$ of patients at 8 hours, $65 \%$ at 12 hours, $55 \%$ at 18 hours, $25 \%$ at 24 hours, $10 \%$ at 36 hours and $30 \%$ at $48 \%$ hours complained of nausea only. Most of patients of group I complained of nausea, retching and even vomiting. as Table 15 shows that most of the patients of group IV were alert, except $55 \%$ of the patients at PACU, $40 \%$ after 2 hours and $10 \%$ after 4 hours who were quietly awake, only $15 \%$ of them at PACU and 5\% after 2 hours who were asleep but easily aroused. Most of patients of group III were asleep but easily aroused at PACU and after 2 hours (100\% and $65 \%$ respectively), after 8 hours all patients of group III were alert, the difference between four groups regarding sedation score was highly statistically significant. $40 \%$ of group I were asleep for longer duration up to 36 hours post-operative, causing a high statistically significant difference with the other groups.

Table 6: Intra-operative End Tidal CO2 (ETCO2) among the studied groups.

\begin{tabular}{|c|c|c|c|c|c|c|}
\hline Variables & $\begin{array}{l}\text { Group I } \\
\mathrm{N}=\mathbf{2 0}\end{array}$ & $\begin{array}{l}\text { Group II } \\
\mathrm{N}=\mathbf{2 0}\end{array}$ & $\begin{array}{l}\text { Group III } \\
\mathrm{N}=20\end{array}$ & $\begin{array}{l}\text { Group IV } \\
\mathrm{N}=20\end{array}$ & F test & P-value \\
\hline $\begin{array}{c}\text { Intra-operative } \mathrm{ETCO}_{2} \text { at skin incision } \\
\text { Rang }\end{array}$ & $\begin{array}{c}36.2 \pm 0.82 \\
36-38\end{array}$ & $\begin{array}{c}37.8 \pm 1.88 \\
35-42\end{array}$ & $\begin{array}{c}37.9 \pm 1.87 \\
35-42\end{array}$ & $\begin{array}{c}37.7 \pm 1.9 \\
35-4\end{array}$ & 2.46 & 0.069 \\
\hline $\begin{array}{c}\text { Intra-operative } \mathrm{ETCO}_{2} \text { at } 5 \mathrm{~min} \\
\text { Mean } \pm \mathrm{SD} \\
\text { Range }\end{array}$ & $\begin{array}{c}35.4 \pm 2.01 \\
35-38\end{array}$ & $\begin{array}{c}36.6 \pm 1.31 \\
35-39\end{array}$ & $\begin{array}{c}36.6 \pm 1.36 \\
35-39\end{array}$ & $\begin{array}{c}36.6 \pm 1.31 \\
35-39\end{array}$ & 2.01 & 0.08 \\
\hline $\begin{array}{c}\text { Intra-operative } \mathrm{ETCO}_{2} \text { at } 10 \mathrm{~min} \\
\text { Mean } \pm \mathrm{SD} \text { Range }\end{array}$ & $\begin{array}{c}36.2 \pm 0.77 \\
35-37\end{array}$ & $\begin{array}{c}36.6 \pm 1.27 \\
35-39\end{array}$ & $\begin{array}{c}36.6 \pm 1.27 \\
35-39\end{array}$ & $\begin{array}{c}36.7 \pm 1.27 \\
35-39\end{array}$ & 0.65 & 0.657 \\
\hline $\begin{array}{c}\text { Intra-operative } \mathrm{ETCO}_{2} \text { at } 15 \mathrm{~min} \\
\text { Mean } \pm \mathrm{SD} \text { Range }\end{array}$ & $\begin{array}{c}35.9 \pm 1.3 \\
34-38\end{array}$ & $\begin{array}{c}36.6 \pm 1.27 \\
35-39\end{array}$ & $\begin{array}{c}36.6 \pm 1.32 \\
35-39\end{array}$ & $\begin{array}{c}36.7 \pm 1.28 \\
35-39\end{array}$ & 2.85 & 0.101 \\
\hline $\begin{array}{c}\text { Intra-operative } \mathrm{ETCO}_{2} \text { at } 30 \mathrm{~min} \\
\text { Mean } \pm \mathrm{SD} \text { Range }\end{array}$ & $\begin{array}{l}36 \pm 2.6 \\
32-40\end{array}$ & $\begin{array}{l}36.95 \pm 1.23 \\
35-39\end{array}$ & $\begin{array}{l}37.1 \pm 1.41 \\
35-40\end{array}$ & $\begin{array}{l}36.95 \pm 1.23 \\
35-39\end{array}$ & 1.85 & 0.17 \\
\hline $\begin{array}{c}\text { Intra-operative } \mathrm{ETCO}_{2} \text { at } 30 \mathrm{~min} \\
\text { Mean } \pm \mathrm{SD} \text { Range }\end{array}$ & $\begin{array}{c}36 \pm 2.6 \\
32-40\end{array}$ & $\begin{array}{l}36.95 \pm 1.23 \\
35-39\end{array}$ & $\begin{array}{c}37.1 \pm 1.41 \\
35-40\end{array}$ & $\begin{array}{c}36.95 \pm 1.23 \\
35-39\end{array}$ & 1.85 & 0.17 \\
\hline $\begin{array}{c}\text { Intra-operative } \mathrm{ETCO}_{2} \text { at } 60 \mathrm{~min} \\
\text { Mean } \pm \mathrm{SD} \text { Range }\end{array}$ & $\begin{array}{l}35.8 \pm 2.55 \\
35-38\end{array}$ & $\begin{array}{c}37.2 \pm 1.32 \\
35-39\end{array}$ & $\begin{array}{c}37.2 \pm 1.32 \\
35-39\end{array}$ & $\begin{array}{c}37.1 \pm 1.25 \\
35-39\end{array}$ & 2.25 & 0.08 \\
\hline $\begin{array}{c}\text { Intra-operative } \mathrm{ETCO}_{2} \text { at } 90 \mathrm{~min} \\
\text { Mean } \pm \mathrm{SD} \text { Range }\end{array}$ & $\begin{array}{l}36.7 \pm 1.78 \\
36-40\end{array}$ & $\begin{array}{l}36.7 \pm 1.78 \\
36-40\end{array}$ & $\begin{array}{c}35.9 \pm 0.74 \\
35-39\end{array}$ & $\begin{array}{c}35.8 \pm 0.99 \\
36-39\end{array}$ & 1.76 & 0.089 \\
\hline $\begin{array}{c}\text { Intra-operative ETCO2 at skin closure } \\
\text { Range }\end{array}$ & $\begin{array}{c}38 \pm 1.45 \\
36-40\end{array}$ & $\begin{array}{l}37.4 \pm 1.35 \\
35-40\end{array}$ & $\begin{array}{l}37.5 \pm 1.45 \\
35-40\end{array}$ & $\begin{array}{c}37.4 \pm 1.35 \\
35-40\end{array}$ & 0.94 & 0.643 \\
\hline
\end{tabular}

Table 7: Intra-operative Total Fentanyl Consumption (TFC) among the studied groups.

\begin{tabular}{|c|c|c|c|c|c|c|}
\hline Variables & $\begin{array}{c}\text { Group I } \\
\mathbf{N}=\mathbf{2 0}\end{array}$ & $\begin{array}{c}\text { Group II } \\
\mathbf{N}=20\end{array}$ & $\begin{array}{l}\text { Group III } \\
\mathrm{N}=20\end{array}$ & $\begin{array}{c}\text { Group IV } \\
\mathbf{N}=\mathbf{2 0}\end{array}$ & F test & P-value \\
\hline $\begin{array}{c}\text { Intra-operative TFC at skin incision Mean } \\
\pm \text { SD Rang }\end{array}$ & $\begin{array}{c}195.3 \pm 23.6 \\
150-200\end{array}$ & $\begin{array}{c}170 \pm 25.13 \\
150-200\end{array}$ & $\begin{array}{c}164 \pm 24.58 \\
130-200\end{array}$ & $\begin{array}{c}164 \pm 24.6 \\
130-200\end{array}$ & 7.41 & $<0.001$ \\
\hline $\begin{array}{l}\text { Intra-operative TFC at } 5 \mathrm{~min} \\
\text { Mean } \pm \text { SD Range }\end{array}$ & $\begin{array}{l}190 \pm 20.5 \\
150-200\end{array}$ & $\begin{array}{l}170 \pm 25.1 \\
150-200\end{array}$ & $\begin{array}{c}164 \pm 24.6 \\
130-200\end{array}$ & $\begin{array}{c}164 \pm 24.6 \\
130-200\end{array}$ & 5.38 & $<0.001$ \\
\hline $\begin{array}{c}\text { Intra-operative TFC at } 10 \mathrm{~min} \\
\text { Mean } \pm \text { SD Range }\end{array}$ & $\begin{array}{c}220 \pm 41.03 \\
150-250\end{array}$ & $\begin{array}{l}170 \pm 25.1 \\
150-200\end{array}$ & $\begin{array}{l}164 \pm 24.6 \\
130-200\end{array}$ & $\begin{array}{c}164 \pm 24.6 \\
130-200\end{array}$ & 16.6 & $<0.001$ \\
\hline $\begin{array}{c}\text { Intra-operative TFC at } 15 \mathrm{~min} \\
\text { Mean } \pm \text { SD Range }\end{array}$ & $\begin{array}{c}237.5 \pm 50.9 \\
150-300\end{array}$ & $\begin{array}{l}170 \pm 25.1 \\
150-200\end{array}$ & $\begin{array}{c}164 \pm 24.6 \\
130-200\end{array}$ & $\begin{array}{c}164 \pm 24.6 \\
130-200\end{array}$ & 23.18 & $<0.001$ \\
\hline $\begin{array}{c}\text { Intra-operative TFC at } 30 \mathrm{~min} \\
\text { Mean } \pm \text { SD Range }\end{array}$ & $\begin{array}{l}260 \pm 32.6 \\
200-300\end{array}$ & $\begin{array}{l}170 \pm 25.1 \\
150-200\end{array}$ & $\begin{array}{l}164 \pm 24.6 \\
130-200\end{array}$ & $\begin{array}{l}164 \pm 24.6 \\
130-200\end{array}$ & 53.52 & $<0.001$ \\
\hline
\end{tabular}




\begin{tabular}{|c|c|c|c|c|c|c|c|}
\hline $\begin{array}{c}\text { Intra-operative } \mathrm{TFC} \text { at } 60 \mathrm{~min} \\
\text { Mean } \pm \text { SD Range }\end{array}$ & & $\begin{array}{c}260 \pm 32.6 \\
200-300\end{array}$ & $\begin{array}{l}170 \pm 25.1 \\
150-200\end{array}$ & $\begin{array}{c}164 \pm 24.6 \\
130-200\end{array}$ & $\begin{array}{l}164 \pm 24.6 \\
130-200\end{array}$ & 3.25 & $<0.001$ \\
\hline $\begin{array}{c}\text { Intra-operative TFC at } 90 \mathrm{~min} \\
\text { Mean } \pm \text { SD Range }\end{array}$ & & $\begin{array}{c}283.3 \pm 24.7 \\
250-300\end{array}$ & $\begin{array}{l}166.7 \pm 25 \\
150-200\end{array}$ & $\begin{array}{l}158 \pm 23 \\
130-200\end{array}$ & $\begin{array}{c}160 \pm 23.5 \\
130-200\end{array}$ & 75.5 & $<0.001$ \\
\hline $\begin{array}{c}\text { Intra-operative TFC at skin closure } \\
\pm \text { SD Range }\end{array}$ & Mean & $\begin{array}{c}260 \pm 38.4 \\
200-300\end{array}$ & $\begin{array}{l}170 \pm 25.1 \\
150-200\end{array}$ & $\begin{array}{c}161.5 \pm 23.2 \\
130-200\end{array}$ & $\begin{array}{c}164 \pm 24.6 \\
130-200\end{array}$ & 55.7 & $<0.001$ \\
\hline
\end{tabular}

Table 8: Post-operative Heart Rate (HR) among the studied groups.

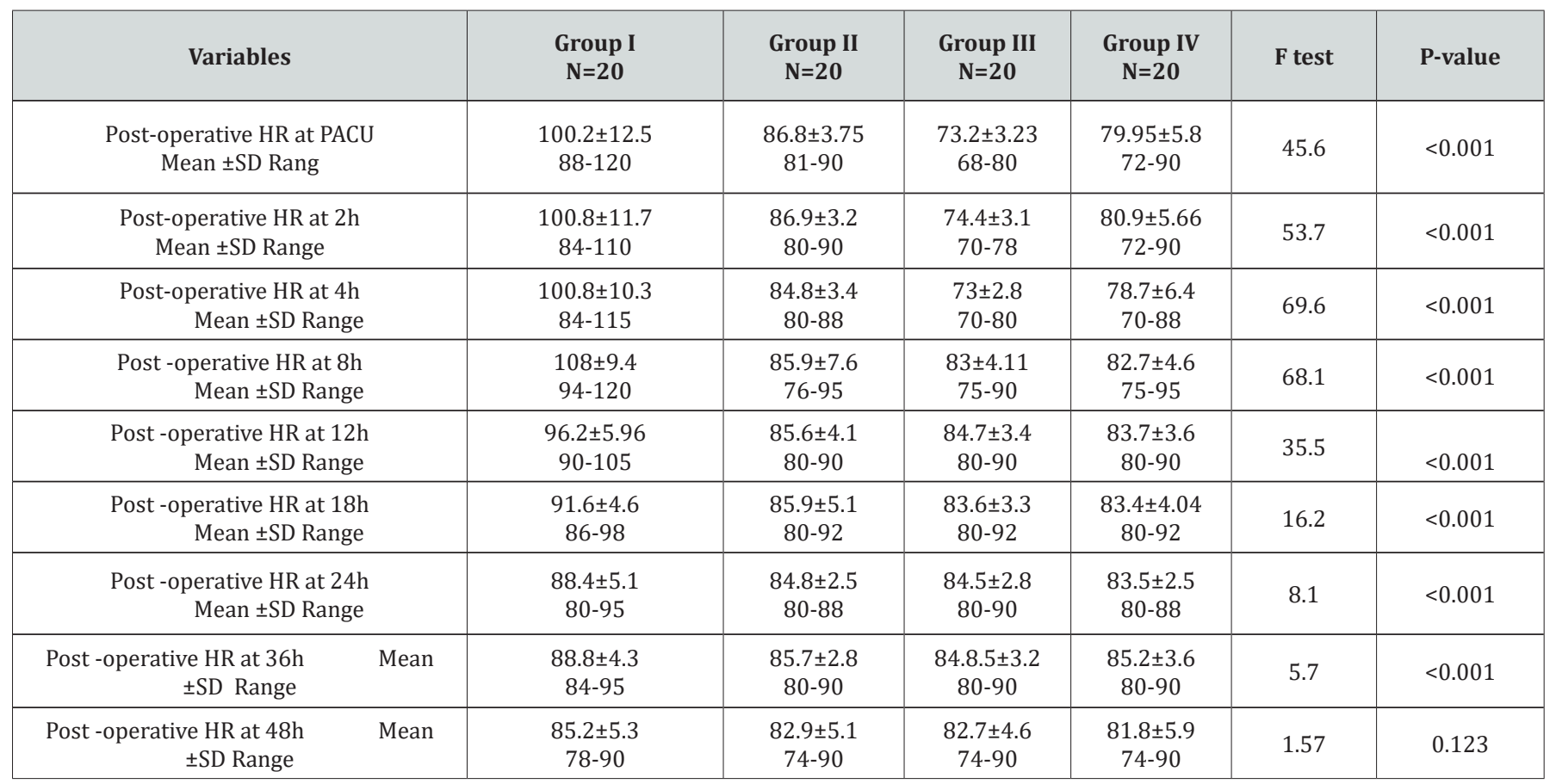

Table 9: Post-operative Mean Arterial blood pressure (MAP) among the studied groups.

\begin{tabular}{|c|c|c|c|c|c|c|}
\hline Variables & $\begin{array}{l}\text { Group I } \\
\mathrm{N}=20\end{array}$ & $\begin{array}{l}\text { Group II } \\
\mathbf{N}=20\end{array}$ & $\begin{array}{l}\text { Group III } \\
\mathbf{N}=20\end{array}$ & $\begin{array}{l}\text { Group IV } \\
\mathbf{N}=20\end{array}$ & F test & P-value \\
\hline $\begin{array}{l}\text { Post-operative MAP at PACU } \\
\text { Mean } \pm \text { SD Rang }\end{array}$ & $\begin{array}{c}90.8 \pm 5.6 \\
82-99\end{array}$ & $\begin{array}{c}73.5 \pm 4.96 \\
68-90\end{array}$ & $\begin{array}{l}72.2 \pm 2.9 \\
68-78\end{array}$ & $\begin{array}{c}76.7 \pm 6.8 \\
70-90\end{array}$ & 53.6 & $<0.001$ \\
\hline $\begin{array}{l}\text { Post-operative MAP at } 2 \mathrm{~h} \\
\text { Mean } \pm \text { SD Range }\end{array}$ & $\begin{array}{l}92 \pm 6.7 \\
80-98\end{array}$ & $\begin{array}{c}70.2 \pm 2.6 \\
68-80\end{array}$ & $\begin{array}{c}69.6 \pm 1.8 \\
65-74\end{array}$ & $\begin{array}{c}72.5 \pm 6.9 \\
67-93\end{array}$ & 89.7 & $<0.001$ \\
\hline $\begin{array}{r}\text { Post-operative MAP at } 4 \mathrm{~h} \\
\text { Mean } \pm \text { SD Range }\end{array}$ & $\begin{array}{l}92 \pm 3.4 \\
86-96\end{array}$ & $\begin{array}{c}75.6 \pm 3.4 \\
70-80\end{array}$ & $\begin{array}{c}73.6 \pm 3.8 \\
66-80\end{array}$ & $\begin{array}{l}72.3 \pm 7.4 \\
57-82\end{array}$ & 73.8 & $<0.001$ \\
\hline $\begin{array}{r}\text { Post-operative MAP at } 8 \mathrm{~h} \\
\text { Mean } \pm \text { SD Range }\end{array}$ & $\begin{array}{c}93.8 \pm 6.4 \\
84-99\end{array}$ & $\begin{array}{c}76.95 \pm 3.03 \\
70-80\end{array}$ & $\begin{array}{l}73.7 \pm 3.6 \\
62-87\end{array}$ & $\begin{array}{l}77.9 \pm 7.6 \\
77-90\end{array}$ & 55.4 & $<0.001$ \\
\hline $\begin{array}{c}\text { Post-operative MAP at } 12 \mathrm{~h} \\
\text { Mean } \pm \text { SD Range }\end{array}$ & $\begin{array}{c}87.6 \pm 3.3 \\
82-91\end{array}$ & $\begin{array}{c}77.8 \pm 4.7 \\
60-82\end{array}$ & $\begin{array}{c}72.9 \pm 2.8 \\
60-88\end{array}$ & $\begin{array}{c}79.3 \pm 8.8 \\
73-82\end{array}$ & 27.6 & $<0.001$ \\
\hline $\begin{array}{c}\text { Post-operative MAP at } 18 \mathrm{~h} \\
\text { Mean } \pm \text { SD Range }\end{array}$ & $\begin{array}{c}84.8 \pm 3.6 \\
78-88\end{array}$ & $\begin{array}{c}76.9 \pm 3.3 \\
68-80\end{array}$ & $\begin{array}{l}72.6 \pm 3.6 \\
58-82\end{array}$ & $\begin{array}{l}77 \pm 7.4 \\
70-87\end{array}$ & $\begin{array}{l}77 \pm 7.4 \\
70-87\end{array}$ & $<0.001$ \\
\hline $\begin{array}{c}\text { Post-operative MAP at } 24 \mathrm{~h} \\
\text { Mean } \pm \text { SD Range }\end{array}$ & $\begin{array}{c}84.8 \pm 4.8 \\
79-92\end{array}$ & $\begin{array}{l}78.9 \pm 3.5 \\
72-86\end{array}$ & $\begin{array}{l}77.9 \pm 2.8 \\
72-86\end{array}$ & $\begin{array}{c}78.2 \pm 2.9 \\
72-86\end{array}$ & 16.8 & $<0.001$ \\
\hline $\begin{array}{c}\text { Post-operative MAP at } 36 \mathrm{~h} \\
\pm \text { SD Range }\end{array}$ & $\begin{array}{c}83.2 \pm 2.1 \\
80-86\end{array}$ & $\begin{array}{l}79.5 \pm 1.8 \\
76-84\end{array}$ & $\begin{array}{l}80.4 \pm 3.2 \\
76-84\end{array}$ & $\begin{array}{l}79.3 \pm 2.02 \\
74-84\end{array}$ & 12.3 & $<0.001$ \\
\hline $\begin{array}{c}\text { Post -operative MAP at } 48 \mathrm{~h} \quad \text { Mean } \\
\pm \text { SD Range }\end{array}$ & $\begin{array}{c}81 \pm 3.7 \\
78-88\end{array}$ & $\begin{array}{l}77.1 \pm 2.7 \\
70-80\end{array}$ & $\begin{array}{l}77.9 \pm 3.6 \\
70-87\end{array}$ & $\begin{array}{l}77.2 \pm 3.4 \\
70-87\end{array}$ & 5.9 & $<0.001$ \\
\hline
\end{tabular}


Table 10: Post-operative Pulsed Oxygen Saturation (SPO2) among the studied groups.

\begin{tabular}{|c|c|c|c|c|c|c|}
\hline Variables & $\begin{array}{c}\text { Group I } \\
\mathrm{N}=\mathbf{2 0}\end{array}$ & $\begin{array}{c}\text { Group II } \\
\mathrm{N}=20\end{array}$ & $\begin{array}{l}\text { Group III } \\
\mathrm{N}=\mathbf{2 0}\end{array}$ & $\begin{array}{c}\text { Group IV } \\
\mathrm{N}=20\end{array}$ & $F$ test & P-value \\
\hline $\begin{array}{c}\text { Post-operative } \mathrm{SPO}_{2} \text { at } \mathrm{PACU} \\
\text { Mean } \pm \mathrm{SD} \text { Rang }\end{array}$ & $\begin{array}{c}97.4 \pm 1.23 \\
96-99\end{array}$ & $\begin{array}{c}98.5 \pm 0.69 \\
98-100\end{array}$ & $\begin{array}{l}99.1 \pm 1.11 \\
96-100\end{array}$ & $\begin{array}{l}99.2 \pm 1.13 \\
96-100\end{array}$ & 11.7 & $<0.001$ \\
\hline $\begin{array}{l}\text { Post-operative } \mathrm{SPO}_{2} \text { at } 2 \mathrm{~h} \\
\text { Mean } \pm \mathrm{SD} \text { Range }\end{array}$ & $\begin{array}{c}95.8 \pm 1.4 \\
94-98\end{array}$ & $\begin{array}{c}98.5 \pm 0.69 \\
98-100\end{array}$ & $\begin{array}{l}98.95 \pm 1.31 \\
95-100\end{array}$ & $\begin{array}{c}99.1 \pm 1.31 \\
95-100\end{array}$ & 32.7 & $<0.001$ \\
\hline $\begin{array}{c}\text { Post-operative } \mathrm{SPO}_{2} \text { at } 4 \mathrm{~h} \\
\text { Mean } \pm \mathrm{SD} \text { Range }\end{array}$ & $\begin{array}{l}95.8 \pm 0.77 \\
95-97\end{array}$ & $\begin{array}{c}95.8 \pm 0.77 \\
95-97\end{array}$ & $\begin{array}{c}97.5 \pm 0.81 \\
96-98\end{array}$ & $\begin{array}{c}97.3 \pm 0.99 \\
96-100\end{array}$ & 35.9 & $<0.001$ \\
\hline $\begin{array}{c}\text { Post -operative } \mathrm{SPO}_{2} \text { at } 8 \mathrm{~h} \\
\text { Mean } \pm \mathrm{SD} \text { Range }\end{array}$ & $\begin{array}{l}92.8 \pm 2.4 \\
90-96\end{array}$ & $\begin{array}{c}98.5 \pm 0.94 \\
98-99\end{array}$ & $\begin{array}{l}98.5 \pm 0.94 \\
98-99\end{array}$ & $\begin{array}{c}97.2 \pm 1.18 \\
95-99\end{array}$ & 64.4 & $<0.001$ \\
\hline $\begin{array}{c}\text { Post -operative } \mathrm{SPO}_{2} \text { at } 12 \mathrm{~h} \\
\text { Mean } \pm \mathrm{SD} \text { Range }\end{array}$ & $\begin{array}{l}92.4 \pm 1.53 \\
90-94\end{array}$ & $\begin{array}{c}98.5 \pm 0.67 \\
98-100\end{array}$ & $\begin{array}{c}97.6 \pm 0.6 \\
96-98\end{array}$ & $\begin{array}{c}97.7 \pm 0.67 \\
96-99\end{array}$ & 196.7 & $<0.001$ \\
\hline $\begin{array}{c}\text { Post -operative } \mathrm{SPO}_{2} \text { at } 18 \mathrm{~h} \\
\text { Mean } \pm \mathrm{SD} \text { Range }\end{array}$ & $\begin{array}{l}91.2 \pm 1.01 \\
90-92\end{array}$ & $\begin{array}{c}98.7 \pm 0.76 \\
98-100\end{array}$ & $\begin{array}{c}97.6 \pm 0.67 \\
97-99\end{array}$ & $\begin{array}{c}97.7 \pm 0.75 \\
97-99\end{array}$ & 353.9 & $<0.001$ \\
\hline $\begin{array}{c}\text { Post -operative } \mathrm{SPO}_{2} \text { at } 24 \mathrm{~h} \\
\text { Mean } \pm \mathrm{SD} \text { Range }\end{array}$ & $\begin{array}{l}94.2 \pm 1.01 \\
93-96\end{array}$ & $\begin{array}{c}98.1 \pm 0.85 \\
95-99\end{array}$ & $\begin{array}{l}98.1 \pm 0.85 \\
95-9\end{array}$ & $\begin{array}{l}98.1 \pm 0.83 \\
95-99\end{array}$ & 95.8 & $<0.001$ \\
\hline $\begin{array}{c}\text { Post -operative SPO2 at } 36 \mathrm{~h} \\
\text { Range }\end{array}$ & $\begin{array}{l}92.4 \pm 0.82 \\
92-94\end{array}$ & $\begin{array}{c}96.9 \pm 1.12 \\
96-99\end{array}$ & $\begin{array}{l}96.9 \pm 1.12 \\
96-99\end{array}$ & $\begin{array}{c}96.9 \pm 1.12 \\
96-99\end{array}$ & 91.3 & $<0.001$ \\
\hline $\begin{array}{c}\text { Post -operative } \mathrm{SPO}_{2} \text { at } 48 \mathrm{~h} \\
\text { Range }\end{array}$ & $\begin{array}{l}93.4 \pm 0.82 \\
92-94\end{array}$ & $\begin{array}{c}96.5 \pm 0.69 \\
96-98\end{array}$ & $\begin{array}{l}96.5 \pm 0.69 \\
96-98\end{array}$ & $\begin{array}{c}96.5 \pm 0.69 \\
96-98\end{array}$ & 91.9 & $<0.001$ \\
\hline
\end{tabular}

Table 11: Post-operative Visual Analogue Scale (VAS) at rest among the studied groups.

\begin{tabular}{|c|c|c|c|c|c|c|}
\hline Variables & $\begin{array}{c}\text { Group I } \\
\mathrm{N}=20\end{array}$ & $\begin{array}{l}\text { Group II } \\
\mathrm{N}=\mathbf{2 0}\end{array}$ & $\begin{array}{c}\text { Group III } \\
\mathrm{N}=20\end{array}$ & $\begin{array}{c}\text { Group IV } \\
\mathrm{N}=\mathbf{2 0}\end{array}$ & F test & P-value \\
\hline $\begin{array}{l}\text { Post-operative VAS at PACU } \\
\text { Mean } \pm \text { SD Rang }\end{array}$ & $\begin{array}{c}19.4 \pm 3.03 \\
15-24\end{array}$ & $\begin{array}{c}18.5 \pm 2.3 \\
14-24\end{array}$ & $\begin{array}{c}7.9 \pm 6.31 \\
0-15\end{array}$ & $\begin{array}{c}8.2 \pm 6.22 \\
0-15\end{array}$ & 34.7 & $<0.001$ \\
\hline $\begin{array}{l}\text { Post-operative VAS at } 2 \mathrm{~h} \\
\text { Mean } \pm \text { SD Range }\end{array}$ & $\begin{array}{c}27.2 \pm 5.7 \\
20-33\end{array}$ & $\begin{array}{c}22.7 \pm 3.5 \\
18-30\end{array}$ & $\begin{array}{c}10.95 \pm 6.3 \\
0-20\end{array}$ & $\begin{array}{c}10.1 \pm 7.3 \\
0-20\end{array}$ & 41.7 & $<0.001$ \\
\hline $\begin{array}{r}\text { Post-operative VAS at } 4 \mathrm{~h} \\
\text { Mean } \pm \text { SD Range }\end{array}$ & $\begin{array}{c}30.4 \pm 5.4 \\
24-40\end{array}$ & $\begin{array}{l}24.95 \pm 3.6 \\
20-34\end{array}$ & $\begin{array}{c}17.3 \pm 4.8 \\
10-23\end{array}$ & $\begin{array}{c}17.3 \pm 5.9 \\
5-23\end{array}$ & 32.5 & $<0.001$ \\
\hline $\begin{array}{r}\text { Post -operative VAS at } 8 \mathrm{~h} \\
\text { Mean } \pm \text { SD Range }\end{array}$ & $\begin{array}{c}30.4 \pm 5.4 \\
22-37\end{array}$ & $\begin{array}{c}28.1 \pm 3.9 \\
23-37\end{array}$ & $\begin{array}{c}23.5 \pm 2.8 \\
20-29\end{array}$ & $\begin{array}{c}23.2 \pm 5.3 \\
10-30\end{array}$ & 12.4 & $<0.001$ \\
\hline $\begin{array}{c}\text { Post -operative VAS at } 12 \mathrm{~h} \\
\text { Mean } \pm \text { SD Range }\end{array}$ & $\begin{array}{c}37.4 \pm 7.6 \\
24-45\end{array}$ & $\begin{array}{c}36 \pm 3.67 \\
30-43\end{array}$ & $\begin{array}{c}24.95 \pm 3.6 \\
20-30\end{array}$ & $\begin{array}{c}23.7 \pm 3.03 \\
20-29\end{array}$ & 44.7 & $<0.001$ \\
\hline $\begin{array}{c}\text { Post -operative VAS at } 18 \mathrm{~h} \\
\text { Mean } \pm \text { SD Range }\end{array}$ & $\begin{array}{c}44.8 \pm 5.3 \\
40-52 \\
\end{array}$ & $\begin{array}{c}44.7 \pm 3.6 \\
40-52\end{array}$ & $\begin{array}{c}25.6 \pm 3.2 \\
20-30\end{array}$ & $\begin{array}{c}25.2 \pm 4.2 \\
20-35\end{array}$ & 146.2 & $<0.001$ \\
\hline $\begin{array}{c}\text { Post -operative VAS at } 24 \mathrm{~h} \\
\text { Mean } \pm \text { SD Range }\end{array}$ & $\begin{array}{c}54.1 \pm 3.8 \\
50-60\end{array}$ & $\begin{array}{c}49.6 \pm 5.1 \\
44-56\end{array}$ & $\begin{array}{c}29.1 \pm 2.8 \\
25-37\end{array}$ & $\begin{array}{c}28.7 \pm 3.03 \\
25-37\end{array}$ & 244.1 & $<0.001$ \\
\hline $\begin{array}{l}\text { Post -operative VAS at } 36 \mathrm{~h} \\
\text { Mean } \pm \text { SD Range }\end{array}$ & $\begin{array}{l}45 \pm 4.2 \\
40-50\end{array}$ & $\begin{array}{c}39.1 \pm 2.99 \\
34-45\end{array}$ & $\begin{array}{c}10.95 \pm 6.4 \\
0-20\end{array}$ & $\begin{array}{c}15.3 \pm 8.6 \\
0-35\end{array}$ & 163 & $<0.001$ \\
\hline $\begin{array}{l}\text { Post -operative VAS at } 48 \mathrm{~h} \\
\text { Mean } \pm \text { SD Range }\end{array}$ & $\begin{array}{c}30.8 \pm 2.2 \\
28-35\end{array}$ & $\begin{array}{c}18.6 \pm 2.8 \\
14-25\end{array}$ & $\begin{array}{c}7.9 \pm 6.3 \\
0-15\end{array}$ & $\begin{array}{c}11.5 \pm 8.3 \\
0-30\end{array}$ & 66.1 & $<0.001$ \\
\hline
\end{tabular}

Table 12: Post-operative Visual Analogue Scale (VAS) on coughing among the studied groups.

\begin{tabular}{|c|c|c|c|c|c|c|}
\hline Variables & $\begin{array}{c}\text { Group I } \\
\mathrm{N}=20\end{array}$ & $\begin{array}{c}\text { Group II } \\
\mathrm{N}=20\end{array}$ & $\begin{array}{c}\text { Group III } \\
\mathrm{N}=20\end{array}$ & $\begin{array}{c}\text { Group IV } \\
\mathrm{N}=20\end{array}$ & F test & P-value \\
\hline $\begin{array}{l}\text { Post-operative VAS at PACU } \\
\text { Mean } \pm \text { SD Rang }\end{array}$ & $\begin{array}{c}29 \pm 5.2 \\
20-35\end{array}$ & $\begin{array}{c}24.5 \pm 2.3 \\
20-29\end{array}$ & $\begin{array}{l}14.9 \pm 5.95 \\
5-20\end{array}$ & $\begin{array}{c}14.9 \pm 6.4 \\
5-20\end{array}$ & 38.3 & $<0.001$ \\
\hline $\begin{array}{l}\text { Post-operative VAS at } 2 \mathrm{~h} \\
\text { Mean } \pm \text { SD Range }\end{array}$ & $\begin{array}{c}41.6 \pm 5.1 \\
35-50\end{array}$ & $\begin{array}{c}31 \pm 3.5 \\
25-37\end{array}$ & $\begin{array}{c}18.2 \pm 5.3 \\
10-25\end{array}$ & $\begin{array}{c}18.2 \pm 6.1 \\
5-25\end{array}$ & 99.5 & $<0.001$ \\
\hline $\begin{array}{r}\text { Post-operative VAS at } 4 \mathrm{~h} \\
\text { Mean } \pm \text { SD Range }\end{array}$ & $\begin{array}{c}48.6 \pm 6.9 \\
38-58\end{array}$ & $\begin{array}{c}37.5 \pm 1.6 \\
34-40\end{array}$ & $\begin{array}{c}24.8 \pm 3.3 \\
20-30\end{array}$ & $\begin{array}{c}28.1 \pm 5.3 \\
12-35\end{array}$ & 102.1 & $<0.001$ \\
\hline
\end{tabular}




\begin{tabular}{|c|c|c|c|c|c|c|c|}
\hline \multicolumn{2}{|c|}{$\begin{array}{r}\text { Post -operative VAS at } 8 \mathrm{~h} \\
\text { Mean } \pm \text { SD Range }\end{array}$} & $\begin{array}{c}46 \pm 3.9 \\
40-50\end{array}$ & $\begin{array}{c}44.4 \pm 3.9 \\
37-52\end{array}$ & $\begin{array}{c}34.5 \pm 3.6 \\
30-40\end{array}$ & $\begin{array}{c}33.95 \pm 6.3 \\
18-40\end{array}$ & 12.4 & $<0.001$ \\
\hline \multicolumn{2}{|c|}{$\begin{array}{c}\text { Post -operative VAS at } 12 \mathrm{~h} \\
\text { Mean } \pm \text { SD Range }\end{array}$} & $\begin{array}{c}53.4 \pm 5.6 \\
47-60\end{array}$ & $\begin{array}{c}53.6 \pm 5.6 \\
40-60\end{array}$ & $\begin{array}{c}35.9 \pm 2.6 \\
30-42\end{array}$ & $\begin{array}{c}35.2 \pm 3.5 \\
29-42\end{array}$ & 39.7 & $<0.001$ \\
\hline \multicolumn{2}{|c|}{$\begin{array}{c}\text { Post -operative VAS at } 18 \mathrm{~h} \\
\text { Mean } \pm \text { SD Range }\end{array}$} & $\begin{array}{c}62.8 \pm 7.7 \\
50-72\end{array}$ & $\begin{array}{c}63.3 \pm 5.02 \\
52-70\end{array}$ & $\begin{array}{c}42.6 \pm 3.2 \\
27-49\end{array}$ & $\begin{array}{c}44.3 \pm 5.96 \\
40-50\end{array}$ & 103.6 & $<0.001$ \\
\hline \multicolumn{2}{|c|}{$\begin{array}{c}\text { Post -operative VAS at } 24 \mathrm{~h} \\
\text { Mean } \pm \text { SD Range }\end{array}$} & $\begin{array}{c}72 \pm 8.5 \\
60-81\end{array}$ & $\begin{array}{c}72.1 \pm 4.8 \\
65-80\end{array}$ & $\begin{array}{c}46.3 \pm 2.3 \\
30-55\end{array}$ & $\begin{array}{c}51.5 \pm 6.98 \\
48-54\end{array}$ & 78.1 & $<0.001$ \\
\hline $\begin{array}{r}\text { Post -operative VAS at } 36 \mathrm{~h} \\
\text { Range }\end{array}$ & Mean \pm SD & $\begin{array}{c}62.8 \pm 10.7 \\
50-79\end{array}$ & $\begin{array}{c}53.1 \pm 7.5 \\
44-65\end{array}$ & $\begin{array}{c}18.5 \pm 5.4 \\
10-25\end{array}$ & $\begin{array}{c}18.9 \pm 4.6 \\
10-25\end{array}$ & 100.2 & $<0.001$ \\
\hline $\begin{array}{r}\text { Post -operative VAS at } 48 \mathrm{~h} \\
\text { Range }\end{array}$ & Mean \pm SD & $\begin{array}{c}43.8 \pm 3.8 \\
40-50\end{array}$ & $\begin{array}{c}34.6 \pm 9.6 \\
24-50\end{array}$ & $\begin{array}{c}14.9 \pm 5.95 \\
5-20\end{array}$ & $\begin{array}{c}15.1 \pm 6.3 \\
5-20\end{array}$ & 191.5 & $<0.001$ \\
\hline
\end{tabular}

Table 13: Post-operative Total Morphine Consumption (TMC) among the studied groups and Post-operative Time to First request for Analgesia (TFA) among the studied groups.

\begin{tabular}{|c|c|c|c|c|c|c|}
\hline Variables & $\begin{array}{l}\text { Group I } \\
\mathrm{N}=\mathbf{2 0}\end{array}$ & $\begin{array}{c}\text { Group II } \\
\mathbf{N}=\mathbf{2 0}\end{array}$ & $\begin{array}{l}\text { Group III } \\
\mathbf{N}=\mathbf{2 0}\end{array}$ & $\begin{array}{l}\text { Group IV } \\
\mathbf{N}=\mathbf{2 0}\end{array}$ & F test & P-value \\
\hline $\begin{array}{c}\text { Post-operative TMC } \backslash \mathrm{mg} \text { at PACU Mean } \\
\pm \text { SD Rang }\end{array}$ & $\begin{array}{c}4 \pm 0.0 \\
4-4\end{array}$ & $\begin{array}{c}4 \pm 0.0 \\
4\end{array}$ & $\begin{array}{c}4 \pm 0.0 \\
4-4\end{array}$ & $\begin{array}{c}4 \pm 0.0 \\
4-4\end{array}$ & & \\
\hline $\begin{array}{c}\text { Post-operative TMC } \backslash \text { mg at } 2 \mathrm{~h} \text { Mean } \pm \mathrm{SD} \\
\text { Range }\end{array}$ & $\begin{array}{c}7.2 \pm 2.1 \\
4-10\end{array}$ & $\begin{array}{l}5.2 \pm 1.01 \\
4-6\end{array}$ & $\begin{array}{c}4 \pm 0.0 \\
4-4\end{array}$ & $\begin{array}{c}4 \pm 0.0 \\
4-4\end{array}$ & 33.5 & $<0.001$ \\
\hline $\begin{array}{c}\text { Post-operative TMC } \backslash \mathrm{mg} \text { at } 4 \mathrm{~h} \text { Mean } \\
\pm \text { SD Range }\end{array}$ & $\begin{array}{c}10.8 \pm 2.1 \\
8-14\end{array}$ & $\begin{array}{c}6.9 \pm 1.02 \\
6-8\end{array}$ & $\begin{array}{c}4.3 \pm 0.73 \\
4-6\end{array}$ & $\begin{array}{c}4.9 \pm 1.02 \\
4-6\end{array}$ & 98.1 & $<0.001$ \\
\hline $\begin{array}{c}\text { Post -operative TMC } \backslash \mathrm{mg} \text { at } 8 \mathrm{~h} \text { Mean } \\
\pm \text { SD Range }\end{array}$ & $\begin{array}{c}15.2 \pm 2.9 \\
12-20\end{array}$ & $\begin{array}{c}8.8 \pm 1.01 \\
8-10\end{array}$ & $\begin{array}{c}6 \pm 0.0 \\
6\end{array}$ & $\begin{array}{c}6 \pm 0.0 \\
6\end{array}$ & 172 & $<0.001$ \\
\hline $\begin{array}{c}\text { Post -operative TMC } \backslash \text { mg at } 12 \mathrm{~h} \text { Mean } \\
\pm \text { SD Range }\end{array}$ & $\begin{array}{c}19.2 \pm 3.1 \\
16-24\end{array}$ & $\begin{array}{c}10.6 \pm 1.6 \\
8-12\end{array}$ & $\begin{array}{c}6.3 \pm 0.73 \\
6-8\end{array}$ & $\begin{array}{c}6.9 \pm 1.02 \\
6-8\end{array}$ & 222 & $<0.001$ \\
\hline $\begin{array}{l}\text { Post -operative TMC } \backslash \text { mg at } 18 \mathrm{~h} \\
\text { Mean } \pm \text { SD Range }\end{array}$ & $\begin{array}{l}23.2 \pm 3.3 \\
20-28\end{array}$ & $\begin{array}{c}12.6 \pm 1.2 \\
10-14\end{array}$ & $\begin{array}{c}7.6 \pm 0.73 \\
6-8\end{array}$ & $\begin{array}{c}7.8 \pm 0.62 \\
6-8\end{array}$ & 308.5 & $<0.001$ \\
\hline $\begin{array}{c}\text { Post -operative TMC } \backslash \text { mg at } 24 \mathrm{~h} \text { Mean } \\
\pm \text { SD Range }\end{array}$ & $\begin{array}{l}27.6 \pm 3.98 \\
24-34\end{array}$ & $\begin{array}{c}14.6 \pm 1.3 \\
12-16\end{array}$ & $\begin{array}{c}8 \pm 1.12 \\
6-10\end{array}$ & $\begin{array}{c}8.7 \pm 1.3 \\
6-10\end{array}$ & 318.8 & $<0.001$ \\
\hline $\begin{array}{c}\text { Post -operative TMC \mg at } 36 \mathrm{~h} \quad \text { Mean } \\
\pm \text { SD Range }\end{array}$ & $\begin{array}{l}30.8 \pm 3.7 \\
26-36\end{array}$ & $\begin{array}{c}15.9 \pm 1.8 \\
12-18\end{array}$ & $\begin{array}{c}8 \pm 1.12 \\
6-10\end{array}$ & $\begin{array}{c}8.7 \pm 1.3 \\
6-10\end{array}$ & 433.2 & $<0.001$ \\
\hline $\begin{array}{c}\text { Post -operative TMC } \backslash \mathrm{mg} \text { at } 48 \mathrm{~h} \text { Mean } \\
\pm \text { SD Range }\end{array}$ & $\begin{array}{c}33.2 \pm 3.8 \\
28-38\end{array}$ & $\begin{array}{c}17.2 \pm 2.6 \\
12-20\end{array}$ & $\begin{array}{c}8 \pm 1.12 \\
6-10\end{array}$ & $\begin{array}{c}8.7 \pm 1.3 \\
6-10\end{array}$ & 435.5 & $<0.001$ \\
\hline $\begin{array}{l}\text { Post-operative TFA \min } \\
\text { Mean } \pm \text { SD Range }\end{array}$ & $\begin{array}{c}24 \pm 9.95 \\
15-40\end{array}$ & $\begin{array}{c}146.75 \pm 17.6 \\
125-190\end{array}$ & $\begin{array}{l}428.8 \pm 60.2 \\
260-500\end{array}$ & $\begin{array}{c}337.5 \pm 85.1 \\
120-480\end{array}$ & 16.22 & $<0.001$ \\
\hline
\end{tabular}

Table 14: Post-operative nausea and vomiting (PONV) among the studied groups.

\begin{tabular}{|c|c|c|c|c|c|c|c|c|c|c|}
\hline \multirow{2}{*}{ Variables } & \multicolumn{2}{|c|}{$\begin{array}{c}\text { Group I } \\
\mathrm{N}=20\end{array}$} & \multicolumn{2}{|c|}{$\begin{array}{c}\text { Group II } \\
\mathbf{N}=\mathbf{2 0}\end{array}$} & \multicolumn{2}{|c|}{$\begin{array}{c}\text { Group III } \\
\mathbf{N}=\mathbf{2 0}\end{array}$} & \multicolumn{2}{|c|}{$\begin{array}{c}\text { Group IV } \\
\mathbf{N}=\mathbf{2 0}\end{array}$} & \multirow{2}{*}{$\mathrm{X}^{2}$ test } & \multirow{2}{*}{ P-value } \\
\hline & $\mathbf{N}$ & $\%$ & $\mathbf{N}$ & $\%$ & $\mathbf{N}$ & $\%$ & $\mathbf{N}$ & $\%$ & & \\
\hline PONV at PACU: None & 4 & 20 & 15 & 75 & 20 & 100 & 20 & 100 & \multirow{4}{*}{49.6} & \multirow{4}{*}{$<0.001$} \\
\hline Nausea & 8 & 40 & 5 & 25 & 0 & 0 & 0 & 0 & & \\
\hline Retching & 4 & 20 & 0 & 0 & 0 & 0 & 0 & & & \\
\hline Vomiting & 4 & 20 & 0 & 0 & 0 & 0 & 0 & & & \\
\hline PONV at $2 \mathrm{~h}: \quad$ None & 4 & 20 & 14 & 70 & 20 & 100 & 20 & 100 & \multirow{3}{*}{48.08} & \multirow{3}{*}{$<0.001$} \\
\hline Nausea & 12 & 60 & 2 & 10 & 0 & 0 & 0 & 0 & & \\
\hline Retching & 4 & 20 & 4 & 20 & 0 & 0 & 0 & 0 & & \\
\hline
\end{tabular}




\begin{tabular}{|c|c|c|c|c|c|c|c|c|c|c|}
\hline PONV at $4 \mathrm{~h}: \quad$ None & 12 & 60 & 14 & 70 & 17 & 85 & 20 & 100 & \multirow{3}{*}{13.58} & \multirow{3}{*}{$<0.001$} \\
\hline Nausea & 4 & 20 & 4 & 20 & 3 & 15 & 0 & 0 & & \\
\hline Retching & 4 & 20 & 2 & 10 & 0 & 0 & 0 & 0 & & \\
\hline PONV at 8h: None & 8 & 40 & 18 & 90 & 17 & 85 & 18 & 90 & \multirow{4}{*}{43.35} & \multirow{4}{*}{$<0.001$} \\
\hline Nausea & 0 & 0 & 2 & 10 & 3 & 15 & 2 & 10 & & \\
\hline Retching & 4 & 20 & 0 & 0 & 0 & 0 & 0 & 0 & & \\
\hline Vomiting & 8 & 40 & 0 & 0 & 0 & 0 & 0 & 0 & & \\
\hline PONV at $12 \mathrm{~h}:$ None & 8 & 40 & 17 & 85 & 7 & 35 & 20 & 100 & \multirow{4}{*}{42.9} & \multirow{4}{*}{$<0.001$} \\
\hline Nausea & 8 & 40 & 2 & 10 & 13 & 65 & 0 & 0 & & \\
\hline Retching & 0 & 0 & 1 & 5 & 0 & 0 & 0 & 0 & & \\
\hline Vomiting & 4 & 20 & 0 & 0 & 0 & 0 & 0 & 0 & & \\
\hline PONV at 18h: None & 12 & 60 & 20 & 100 & 6 & 30 & 20 & 100 & \multirow{4}{*}{61.9} & \multirow{4}{*}{$<0.001$} \\
\hline Nausea & 0 & 0 & 0 & 0 & 11 & 55 & 0 & 0 & & \\
\hline Retching & 4 & 20 & 0 & 0 & 3 & 15 & 0 & 0 & & \\
\hline Vomiting & 4 & 20 & 0 & 0 & 0 & 0 & 0 & 0 & & \\
\hline PONV at $24 \mathrm{~h}:$ None & 12 & 60 & 6 & 30 & 15 & 75 & 14 & 70 & \multirow{4}{*}{27.98} & \multirow{4}{*}{$<0.001$} \\
\hline Nausea & 4 & 20 & 12 & 60 & 5 & 25 & 6 & 30 & & \\
\hline Retching & 4 & 20 & 0 & 0 & 0 & 0 & 0 & 0 & & \\
\hline Vomiting & 0 & 0 & 2 & 10 & 0 & 0 & 0 & 0 & & \\
\hline PONV at 36h: None & 8 & 40 & 20 & 100 & 18 & 90 & 20 & 100 & \multirow{2}{*}{34.3} & \multirow{2}{*}{$<0.001$} \\
\hline Nausea & 12 & 60 & 0 & 0 & 2 & 10 & 0 & 0 & & \\
\hline PONV at 48h: None & 12 & 60 & 20 & 100 & 14 & 70 & 20 & 100 & \multirow{2}{*}{17.66} & \multirow{2}{*}{$<0.001$} \\
\hline Nausea & 8 & 40 & 0 & 0 & 6 & 30 & 0 & 0 & & \\
\hline
\end{tabular}

Table 15: Post-operative Sedation Scale among the studied groups.

\begin{tabular}{|c|c|c|c|c|c|c|c|c|c|c|}
\hline \multirow[t]{2}{*}{ Variables } & \multicolumn{2}{|c|}{$\begin{array}{c}\text { Group I } \\
\mathrm{N}=20\end{array}$} & \multicolumn{2}{|c|}{$\begin{array}{c}\text { Group II } \\
\mathrm{N}=\mathbf{2 0}\end{array}$} & \multicolumn{2}{|c|}{$\begin{array}{c}\text { Group III } \\
\mathrm{N}=\mathbf{2 0}\end{array}$} & \multicolumn{2}{|c|}{$\begin{array}{c}\text { Group IV } \\
\mathbf{N}=\mathbf{2 0}\end{array}$} & \multirow[t]{2}{*}{$\mathrm{X}^{2}$ test } & \multirow[t]{2}{*}{ P-value } \\
\hline & $\mathbf{N}$ & $\%$ & $\mathbf{N}$ & $\%$ & $\mathbf{N}$ & $\%$ & $\mathbf{N}$ & $\%$ & & \\
\hline $\begin{array}{c}\text { Sedation at PACU: } \\
\text { Alert }\end{array}$ & 4 & 20 & 3 & 15 & 0 & 0 & 6 & 30 & \multirow{3}{*}{33.03} & \multirow{3}{*}{$<0.001$} \\
\hline Quietly awake & 8 & 40 & 10 & 50 & 0 & 0 & 11 & 55 & & \\
\hline Asleep but easily aroused & 8 & 40 & 7 & 35 & 20 & 100 & 3 & 15 & & \\
\hline Sedation at $2 \mathrm{~h}$ : Alert & 4 & 20 & 2 & 10 & 2 & 10 & 11 & 55 & \multirow{3}{*}{23.8} & \multirow{3}{*}{$<0.001$} \\
\hline Quietly awake & 8 & 40 & 11 & 55 & 5 & 25 & 8 & 40 & & \\
\hline Asleep but easily aroused & 8 & 40 & 7 & 35 & 13 & 65 & 1 & 1 & & \\
\hline Sedation at $4 \mathrm{~h}$ : alert & 4 & 20 & 6 & 30 & 12 & 85 & 18 & 90 & \multirow{3}{*}{26.65} & \multirow{3}{*}{$<0.05$} \\
\hline Quietly awake & 12 & 60 & 11 & 55 & 8 & 15 & 2 & 10 & & \\
\hline Asleep but easily aroused & 4 & 20 & 3 & 15 & 0 & 0 & 0 & 0 & & \\
\hline Sedation at 8h: Alert & 4 & 20 & 12 & 60 & 20 & 100 & 20 & 100 & \multirow{3}{*}{52.58} & \multirow{3}{*}{$<0.001$} \\
\hline Quietly awake & 8 & 40 & 8 & 40 & 0 & 0 & 0 & 0 & & \\
\hline Asleep but easily aroused & 8 & 40 & 0 & 0 & 0 & 0 & 0 & 0 & & \\
\hline
\end{tabular}




\section{Journal of Gynecology and Women's Health}

\begin{tabular}{|c|c|c|c|c|c|c|c|c|c|c|}
\hline Sedation at $12 \mathrm{~h}$ : Alert & 12 & 60 & 12 & 60 & 20 & 100 & 20 & 100 & \multirow{3}{*}{52.23} & \multirow{3}{*}{$<0.001$} \\
\hline Quietly awake & 4 & 20 & 7 & 35 & 0 & 0 & 0 & 0 & & \\
\hline Asleep but easily aroused & 4 & 20 & 1 & 5 & & & & & & \\
\hline Sedation at 18h: Alert & 4 & 20 & 12 & 60 & 20 & 100 & 20 & 100 & \multirow{3}{*}{41.91} & \multirow{3}{*}{$<0.001$} \\
\hline Quietly awake & 16 & 80 & 8 & 40 & 0 & 0 & 0 & 0 & & \\
\hline Asleep but easily aroused & 0 & 0 & 0 & 0 & 0 & 0 & 0 & 0 & & \\
\hline Sedation at $24 \mathrm{~h}$ : Alert & 12 & 60 & 18 & 90 & 20 & 100 & 20 & 100 & \multirow{3}{*}{21.98} & \multirow{3}{*}{$<0.001$} \\
\hline Quietly awake & 4 & 20 & 2 & 10 & 0 & 0 & 0 & 0 & & \\
\hline Asleep but easily aroused & 4 & 20 & 0 & 0 & 0 & 0 & 0 & 0 & & \\
\hline Sedation at 36h: Alert & 4 & 20 & 20 & 100 & 20 & 100 & 20 & 100 & \multirow{3}{*}{60} & \multirow{3}{*}{$<0.001$} \\
\hline Quietly awake & 8 & 40 & 0 & 0 & 0 & 0 & 0 & 0 & & \\
\hline Asleep but easily aroused & 8 & 40 & 0 & 0 & 0 & 0 & 0 & 0 & & \\
\hline Sedation at 48h: Alert & 16 & 20 & 20 & 100 & 20 & 100 & 20 & 100 & \multirow{3}{*}{12.66} & \multirow{3}{*}{$<0.001$} \\
\hline Quietly awake & 4 & 80 & 0 & 0 & 0 & 0 & 0 & 0 & & \\
\hline Asleep but easily aroused & 0 & 0 & 0 & 0 & 0 & 0 & 0 & 0 & & \\
\hline
\end{tabular}

\section{Discussion}

Many of studies done to expand the postoperative painfree period as long as to allow early ambulation, decrease the postoperative morbidity and decrease the hospital stay and cost. These studies include use of neuroaxonal blocks (subarachnoid, epidural and caudal), peripheral nerve blocks (paravertebral, transversus abdominis plexus and local infiltration) and the use of different analgesic medications e.g. patient- controlled analgesia (PCA). Not only the different approaches of peripheral nerve plexuses blockade but also different local anesthetics concentrations and use of additives had played roles in the previous studies. Effective analgesia has shown to reduce postoperative stress response and accelerate recovery from surgery [8].

A multimodal approach to postoperative analgesia after abdominal surgeries is required to block nociceptive transmission from both the abdominal wall incision and from the abdominal viscera .It is known from cadaveric and observational studies that a single shot lumbar TAP block with $20 \mathrm{ml}$ of local anesthetics is effective in blocking the corresponding dermatomes [9].

The analgesic efficacy of the TAP block has been demonstrated in prospective randomized trials compared with placebo, in different surgical procedures such as hysterectomy who also reported that the addition of TAP block to conventional general anesthesia reduces the morphine requirements [10]. The use of ultrasound-guided sensory block of the anterior abdominal wall with local anesthesia for postoperative pain relief is an attractive method because of its simplicity and safety [11].

In our study, results showed that patients who received TAP block with $20 \mathrm{ml}$ bupivacaine on each side even without any additives had significantly reduced morphine consumption at $48 \mathrm{~h}$ post operatively $(17.2 \pm 2.6 \mathrm{mg}$ vs. $33.2 \pm 3.8 \mathrm{mg}$ in patients who not received TAP block, $\mathrm{P}<0.001$ ). Also, Pain scores at rest and on coughing were significantly lower in the TAP block group even without additives especially at PACU up to $4 \mathrm{~h}$ after surgery. The incidence of sedation and PONV were reduced in patients undergoing TAP block, and there were no complications related to the block.

Niraj and colleagues studied the efficacy of unilateral, ultrasound-guided TAP block in patients with an anterolateral abdominal wall incision for open appendicectomy. The results of their study showed that the patients who received TAP block had significantly reduced morphine consumption at $24 \mathrm{~h}$ after surgery. Pain scores at rest and on coughing were significantly lower in the TAP block group [11]. In a study by Carney et al., comparing TAP block with TAP placebo block after elective total abdominal hysterectomy, their results showed that TAP block with ropivacaine reduced postoperative pain scores compared with the placebo block. Total morphine requirements in the first 48 postoperative hours were also reduced ( $55 \pm 17$ vs. $27 \pm 20 \mathrm{mg}$ ). The incidence of sedation was reduced in patients undergoing TAP blockade, and there were no complications related to the block [12].

Another study showed that ultrasound-guided technique enables exact placement of the local anesthetic for TAP blocks. In patients undergoing laparoscopic cholecystectomy under standard general anesthesia, ultrasound-guided TAP block with $15 \mathrm{ml}$ bupivacaine $5 \mathrm{mg} / \mathrm{ml}$ on each side reduced the postoperative morphine consumption [10.5 (7.7) vs. 22.8 (4.3)mg, $\mathrm{P}<0.05$ ] [13]. In a randomized controlled trial, the analgesic efficacy of TAP block after abdominal surgery was studied, and the results 
showed that TAP block reduced pain scores on emergence $(1 \pm 1.4$ vs. $6.6 \pm 2.8, \mathrm{P}<0.05)$, and at all postoperative time points, including at $24 \mathrm{~h}(1.7 \pm 1.7$ vs. $3.1 \pm 1.5, \mathrm{P}<0.05)$. Morphine requirements in the first 24 postoperative hours were also reduced $(21.9 \pm 8.9$ vs. $80.4 \pm 19.2 \mathrm{mg}, \mathrm{P}<0.05$ ) and there were no complications attributable to the TAP block. All TAP patients reported high levels of satisfaction with their postoperative analgesic regimen [14].

Griffiths et al. [15] demonstrated that TAP blockade provided no benefit in addition to multimodal analgesia in women undergoing major gynecological cancer surgery, with inadequate analgesia either at rest ( 39 vs. $22 \%, \mathrm{P}=0.13$ ) or with coughing ( 61 vs. $53 \%, \mathrm{P}=0.54$ ) at $2 \mathrm{~h}$. They found no significant difference in the postoperative morphine consumption between the placebo and the treatment groups at $2 \mathrm{~h}(13.5 \mathrm{vs} .11 .87 \mathrm{mg}, \mathrm{P}=0.53)$ or 24 h (34.0 vs. $36.1 \mathrm{mg}, \mathrm{P}=0.76$ ). They also found no significant differences in the incidence of opioid side effects or patient satisfaction [15].

In our study, we perform TAP block before surgical incision and before induction of anesthesia to assess success of block either by pin prick or by cold sensation. Also, we noticed the analgesic effect of TAP block intra operatively by improvement of hemodynamic measurements like HR and MAP with significantly reduced total fentanyl consumption in TAP block group even without any additives at skin closure at end of surgery $(170 \pm 25.1$ mcg vs $260 \pm 38.4 \mathrm{mcg}$ in patients who not received TAP block, $\mathrm{P}$ $<0.001$ ).

Many articles and case studies have demonstrated the analgesic effect of TAP block when it had been performed at the end of surgery, before emergence from anesthesia [16]. Others have reported the analgesic effect of TAP block when it had been performed after anesthetic induction, before surgical incision [11]. The current study showed that the addition of $8 \mathrm{mg}$ dexamethasone to $20 \mathrm{~mL}$ bupivacaine $0.25 \%$ for TAP block on both sides resulted in prolongation the analgesic effect of TAP block by prolongation TFA with mean time $(356.2 \pm 62.7 \mathrm{~min}$ vs. $146.75 \pm 17.6 \mathrm{~min}$ in most patients of TAP group without any additives) and by reducing TMC at $48 \mathrm{~h}$ post operatively $(8.7 \pm 1.3 \mathrm{mg}$ vs. $17.2 \pm 2.6 \mathrm{mg}$ in patients of TAP group without any additives) and a significant reduction of VAS pain score over the postoperative $48 \mathrm{~h}$ at rest and on coughing than in TAP block group without any additives.

In a study by Movafegh et al. to evaluate the effect of dexamethasone added to lidocaine on the onset and the duration of axillary brachial plexus block, they found that it resulted in a longer sensory (the sensory blockade duration was $242 \pm 76$ vs. $98 \pm 33 \mathrm{~min}$ for the control) and motor block (the motor blockade duration was $310 \pm 81$ vs. $130 \pm 31 \mathrm{~min}$ for the control) [17]. In another study adding $8 \mathrm{mg}$ of dexamethasone to a mixture of lidocaine and bupivacaine for supraclavicular brachial plexus block, there was a significantly faster onset of action and prolonged duration of analgesia in the dexamethasone group than in the control group with no reported complications [18].

In another study, Parrington etal.added $8 \mathrm{mg}$ of dexamethasone to mepivacaine during supraclavicular brachial plexus blockade. The dexamethasone group showed a longer duration of analgesia of 332 versus $228 \mathrm{~min}$ in the control group, whereas the onset times were similar in both groups for sensory and motor blockade [18]. Cummings et al. [19] studied the effect of dexamethasone on the duration of interscalene nerve blocks with ropivacaine or bupivacaine. Dexamethasone significantly prolonged the duration of analgesia of both ropivacaine [11.8 (9.7-13.8) vs. 22.2 (18.028.6)h] and bupivacaine [14.8 (11.8-18.1) vs. $22.4(20.5-29.3) \mathrm{h}]$ [19].

Shrestha and coworkers added $8 \mathrm{mg}$ of dexamethasone to a mixture of lidocaine and bupivacaine for supraclavicular brachial plexus block. Dexamethasone provided a faster onset of action and longer duration of analgesia without any adverse effects [20]. It should be emphasized that a blockade is not produced if steroids are used alone for regional blocks, but steroids may potentiate the action of local anesthetics through modulation of the function of potassium channels in the excitable cells [21]. In contradiction to our results, Tan and colleagues reported failure of IV dexamethasone to potentiate the analgesic effect of intrathecal neostigmine; but, in our study, dexamethasone was not used intravenously other than for TAP block [22].

Another study reported analgesic and antiemetic effect of betamethason during ambulatory surgery [23]. Which was matching with our study in reducing both pain and vomiting in which the group of TAP block with dexamethasone did not complain of nausea nor vomiting post-operatively except $10 \%$ of patients at 8 hours and $30 \%$ at 24 hours who complained of nausea only and the difference between groups was highly statistically significant.

In our prospective, randomized study showed not only the efficacy of TAP block in relieving postoperative pain after lower abdominal surgeries but also extended time of pain relief in dexmedetomidine group more than that with other groups by assessment of VAS during rest and during movements. Also the postoperative first request of morphine was delayed and total morphine consumption through $48 \mathrm{~h}$ was obviously reduced in dexmedetomidine group more than that with other groups. This study demonstrates also the risk reduction for postoperative nausea and vomiting that resulted either from pain or from high doses of morphine consumption.

In our study we used dexmedetomidine by dose of $0.8 \mathrm{ug} / \mathrm{kg}$ that showed prolonged time of analgesia in dexmedetomidine TAP group than bupivacaine alone TAP group and prolonged time of first request for morphine and reduction in total morphine consumption in the dexmedetomidine TAP group. This comes in 
agreement with the later studies. Saadawy and coworkers added dexmedetomidine $(1 \mu \mathrm{g} / \mathrm{kg})$ to bupivacaine for caudal anesthesia in pediatrics; achieving longer analgesia, less rescue analgesic consumption, and improved sleep quality with no adverse clinically relevant side effects [24].

Kanazi et al., demonstrated that dexmedetomidine $(3 \mu \mathrm{g})$ in combination with bupivacaine for spinal anesthesia has been shown to provide a shorter onset to motor blockade and prolongation of motor and sensory block along with preservation of hemodynamics and absence of sedation [5]. Also, dexmedetomidine $(5 \mu \mathrm{g})$ when added to intrathecal bupivacaine during gynecologic surgeries has resulted in a longer sensory and motor block duration as demonstrated by Al-Ghanem and his colleagues [26]. Many studies were done to demonstrate the efficacy of dexmedetomidine in combination with local anesthetics during peripheral nerve and nerve plexus blockade. A study by Esmaoglu et al., mixed dexmedetomidine with levobupivacaine during placement of axillary brachial plexus blockade that resulted in shortening of block onset time and longer block duration resulting in improved postoperative analgesia [27].

Another study by Obayah et al., added dexmedetomidine to bupivacaine during placement of a greater palatine nerve block for cleft palate repair. The addition of dexmedetomidine to bupivacaine provided lower pain scores and prolonged analgesia (approximately 50\%) with no negative effect on hemodynamics when compared with bupivacaine alone [28]. On the other hand, Ozalp et al. have compared dexmedetomidine -ropivacaine mixture to ropivacaine alone in patient controlled interscalene analgesia and they reported similar pain scores in both groups without any advantageous effect of dexemedetomidine [29].

Similar to our finding, many investigators reported that the addition of dexmedetomidine to different types of LA agents in various types of peripheral nerve blocks resulted in prolongation of analgesic effect [30]. On the other hand, Masuki et al. [31] suggested that dexmedetomidine induces vasoconstriction through an action on $\alpha 2$ adrenoceptors in the human forearm and the later might contribute to the longer duration of action [31]. Dexmedetomidine might associate with some side-effect such as hypotension, bradycardia and sedation particularly at higher doses [25]. In our study, we noticed a significant fall in the HR 60 beats /min following the administration of dexmedetomidine opposite to the other groups. This effect persisted for $4 \mathrm{~h}$, but without any hemodynamic instability. The decrease in pulse rate might be related to the post-synaptic activation of central $\alpha 2$ adrenoceptors, leading to decreased sympathetic activity and slower HR [32]. Similar to HR increased sedation was noticed in the first post-operative hour among dexmedetomidine TAP group patients. None of our patients required treatment for the low HR or sedation. The low dose of dexmedetomidine used in our study might be the reason behind the minor adverse events.

\section{Conclusion}

TAP block as a part of a multimodal analgesia regimen provided better analgesia after abdominal hysterectomy operation with lesser incidence of opioid-related side effects (sedation or nausea) compared with systemic analgesia with morphine due to the lower dose of morphine. TAP block as multimodal postoperative analgesia might be an option to facilitate postoperative early ambulation. The addition of dexamethasone $8 \mathrm{mg}$ to bupivacaine $0.25 \%$ for TAP block provides prolongation of the duration of the block and decreases the incidence of postoperative nausea and vomiting that may have a great impact on patient comfort.

Using dexmedetomidine as an additive to bupivacaine in ultrasound-guided TAP block for abdominal hysterectomy surgeries provides prolonged duration of postoperative analgesia, lowered VAS pain scores and reduces supplemental opioid requirements without any major side-effects. Addition of dexmedetomidine to bupivacaine in ultrasound-guided TAP block for abdominal hysterectomy has the upper hand than addition of dexamethasone in prolongation of the duration of the block but addition of dexamethasone is a very good alternative additive especially in relation of cost benefit side and decreases the incidence of postoperative nausea and vomiting.

\section{Limitations}

One limitation of the current study results from the fact that the beneficial effects of dexamethasone could be explained by its systemic absorption. Unfortunately, because another group (perineural bupivacaine + intravenous dexamethasone) was not included in the design of the study, we were unable to answer this question. A second limitation is the inability to assess dexmedetomidine plasma concentration among study patients to determine whether its action was related to systemic absorption or pure local effect. Further studies are needed to determine the safe effective dose of dexmedetomidine and to assess the risk of perineural administration of dexmedetomidine among bigger patients' sample.

\section{References}

1. (1994) International Association for the Study of Pain Task Force on Taxonomy. In: (2 ${ }^{\text {nd }}$ edn), In: Merskey NB (.Ed), Classification of Chronic Pain, IASP Press, USA, pp. 209-214.

2. Ng A, Swami A, Smith G, Davidson AC, Emembolu J, et al. (2002) The analgesic effects of intraperitoneal and incisional bupivacaine with epinephrine after total abdominal hysterectomy. Anesth Analg 95(1): 158-162.

3. Ganai S, Lee KF, Merrill A, Lee MH, Bellantonio S, et al. (2007) Adverse outcomes of geriatric patients undergoing abdominal surgery who are at high risk for delirium. Arch Surg 142(11): 1072-1078.

4. McDonnell JG, O' Donnell BD, Tuite D, Farrell T, Power C, et al (2004) The regional abdominal field infiltration (R.A.F.I.) technique: Computerized Tomographic and anatomical identification of a novel approach to the transversus abdominis neuro-vascular fascial plane. Anesthesiology 101: A899. 
5. Hebbard P, Fujiwara Y, Shibata Y, Royse C (2007) Ultrasound guided transversus abdominis plane (TAP) block. Anaesth Intensive Care 35(4): 616-617.

6. Ammar AS, Mahmoud KM (2012a) Effect of adding dexamethasone to bupivacaine on transversus abdominis plane block for abdominal hysterectomy: A prospective randomized controlled trial. Saudi J Anesth 6(3): 229-33.

7. Rasmussen SB, Saied NN, Bowens CJr, Mercaldo ND, Schildcrout JS, et al. (2013) Duration of upper and lower extremity peripheral nerve blockade is prolonged with dexamethasone when added to ropivacaine: a retrospective database analysis. Pain Med (Malden, Mass) 14(8): 1239-1247.

8. Kehlet H (1989) Surgical stress: the role of pain and analgesia. Br J Anaesth 63(2): 189-195.

9. Tran TM, Ivanusic JJ, Hebbard P, Barrington MJ (2009) Determination of spread of injectate after ultrasound-guided transversus abdominis plane block: A cadaveric study. Br J Anaesth 102(1): 123-127.

10. Carney JJ, McDonnell JG, Ochana A, Bhinder R, Laffey JG, et al. (2008) The transversus abdominis plane block provides effective postoperative analgesia in patients undergoing total abdominal hysterectomy. Anesth Anlg 107(6): 2056-2060.

11. Niraj G, Kelkar A, Fox AJ (2009a) Application of the transverses abdominis plane block in the intensive care unit. Anaesth Intensive Care 37(4): 650-652.

12. Tsui B (2007) Equipment for regional anesthesia in children. Tech Reg Anesth Pain Man 11: 235-246.

13. El-Dawlatly AA, Turkistani A, Kettner SC, Machata A-M, Delvi MB, et al. (2009) Ultrasound-guided transversus abdominis plane block: description of a new technique and comparison with conventional systemic analgesia during laparoscopic cholecystectomy. Br J Anaesth 102(6): 763-767.

14. McDonnell JG, O’Donnell B, Curley G, Heffernan A, Power C, et al. (2007a) The analgesic efficacy of Transvesus Abdominis plane block after abdominal surgery: a prospective randomized controlled trial. Anesth Analg 104(1): 193-197.

15. Griffiths J, Middle JV, Barron FA, Grant SJ, Popham PA, et al. (2010) Transversus abdominis plane block does not provide additional benefit to multimodal analgesia in gynecological cancer surgery. Anesth Analg 111(3): 797-801.

16. French JL, Mccullough J, Bachra P, Bedforth NM (2009) Transversus abdominis plane block for analgesia after Caesarean section in a patient with an intracranial lesion. Int J Obstet Anesth 18(1): 52-54.

17. Movafegh A, Razazian M, Hajimaohamadi F, Meysamie A (2006) Dexamethasone added to lidocaine prolongs axillary brachial plexus blockade. Anesth Analg 102(1): 263-267.

18. Parrington SJ, O’Donnell D, Chan VW, Brown-Shreves D, Subramanyam $\mathrm{R}$, et al. (2010) Dexamethasone added to mebivacaine prolongs the duration of analgesia of after supraclavicular brachial plexus blockade. Reg Anesth Pain Med 35(5): 422-426.
19. Cummings KC, Napierkowski DE, Parra-Sanchez I, Kurz A, Dalton JE et al. (2011) Effect of dexamethasone on the duration of interscalene nerve blocks with ropivacaine or bupivacaine. Br J Anaesth 107(3): 446-453.

20. Shrestha BR, Maharjan SK, Tabedar S (2003) Supraclavicular brachial plexus block with and without dexamethasone -A comparative study. Kathmandu Univ Med J (KUMJ) 1(3): 158-160.

21. Pennington AJ, Kelly JS, Antoni FA (1994) Selective enhancement of an A type potassium current by dexamethasone in a corticotroph cell line. J Neuroendocrinol 6(3): 305-315.

22. Tan PH, Liu K, Peng CH, Yang LC, Lin CR, et al. (2001) The effect of dexamethasone on postoperative pain and emesis after intra-thecal neostigmine. Anesth Analg 92(1): 228-232.

23. Aasboe V, Raeder JC, Groegaard B (1998) Betamethasone reduces postoperative pain and nausea after ambulatory surgery. Anesth Analg 87(2): 319-323.

24. Saadawy I, Boker A, Elshahawy MA, Almazrooa A, Melibary S, et al. (2009) Effect of dexmedetomidine on the characteristics of bupivacaine in a caudal block in pediatrics. Acta Anaesthesiol Scand 53(2): 251-256

25. Kanazi GE, Aouad MT, Jabbour-Khoury SI, Al Jazzar MD, Alameddine MM, et al. (2006) Effect of low-dose dexmedetomidine or clonidine on the characteristics of bupivacaine spinal block. Acta Anaesthesiol Scand 50(2): 222-227.

26. Al-Ghanem SM, Massad IM, Al-Mustafa MM, Al-Zaben KR, Qudaisat IY, et al. (2009) Effect of adding dexmedetomidine versus fentanyl to intrathecal bupivacaine on spinal block characteristics in gynecological procedures: A double blind controlled study. Am J Appl Sci 6: 882-887.

27. Esmaoglu A, Yegenoglu F, Akin A, Turk CY (2010) Dexmedetomidine added to levobupivacaine prolongs axillary brachial plexus block Anesth Analg111(6): 1548-1551.

28. Obayah GM, Refaie A, Aboushanab O, Ibraheem N, Abdelazees M, et al. (2010) Addition of dexmedetomidine to bupivacaine for greater palatine nerve block prolongs postoperative analgesia after cleft palate repair. Eur J Anaesthesiol 27(3): 280-284

29. Ozalp G, Tuncel G, Savli S, Celik A, Doger C, et al. (2006) The analgesic efficacy of dexmedetomidine added to ropivacaine patient controlled interscalene analgesia via the posterior approach. J Anaesth 21: 409412.

30. Rancourt MP, Albert NT, Côté M, Létourneau DR, Bernard PM, et al (2012) Posterior tibial nerve sensory blockade duration prolonged by adding dexmedetomidine to ropivacaine. Anesth Analg 115(4): 958962.

31. Masuki S, Dinenno FA, Joyner MJ, Eisenach JH (2005) Selective alpha2 adrenergic properties of dexmedetomidine over clonidine in the human forearm. J Appl Physiol 99(2): 587-592.

32. Talke P, Lobo E, Brown R (2003) Systemically administered alpha2- agonist-induced peripheral vasoconstriction in humans. Anesthesiology 99(1): 65-70. 
Your next submission with Juniper Publishers will reach you the below assets

- Quality Editorial service

- Swift Peer Review

- Reprints availability

- E-prints Service

- Manuscript Podcast for convenient understanding

- Global attainment for your research

- Manuscript accessibility in different formats ( Pdf, E-pub, Full Tsext, Audio)

- Unceasing customer service

Track the below URL for one-step submission https://juniperpublishers.com/online-submission.php 